\title{
WEYL GROUPS AND THE REGULARITY PROPERTIES OF CERTAIN COMPACT LIE GROUP ACTIONS
}

\author{
ELDAR STRAUME
}

\begin{abstract}
The geometric weight system of a $G$-manifold $X$ (acyclic or spherical) is the nonlinear analogue of the weight system of a linear representation. We study the possible realization of a given $G$-weight pattern, via the interaction between roots, weights and the Weyl group, together with various fixed point results of P. A. Smith type. If the orbit structure is reasonably simple, then the $G$-weight pattern must in fact coincide with that of a simple representation. This in turn implies that $X$ is (orthogonally) modeled on the linear $G$-space, e.g., with the same orbit types. In particular, complete results in this direction are obtained for a certain family of $G$-manifolds, $G$ a classical group. In this family the weight patterns are of 2-parametric type, and it includes essentially all cases where the principal isotropy type is nontrivial. This family also covers many cases with trivial principal isotropy type.
\end{abstract}

1. Introduction. In compact Lie transformation groups, one of the natural problems is the study of orbit structures, namely, for a given group $G$ and space $X$ one investigates how $X$ can be made into a $G$-space. Then it becomes pertinent to find some suitable invariants which, on the one hand, are sufficiently simple to work with, but on the other hand also exert a considerable amount of control of the orbit structure - thus making the investigation of orbit structure into a feasible task. If $X$ is a "classical" space, say, of acyclic, spherical or projective type, then there are natural $G$-linear models to compare with; this gives rise to a comparison principle. Roughly speaking, this expresses one's expectation that if the $G$-action $\varphi$ is not too complicated, then the orbit structure of the $G$-manifold $X$ resembles that of a suitable linear model to a large extent.

One of the main purposes of this paper is to add new evidence to this principle by establishing regularity theorems for smooth actions of classical groups. The basic tools are provided by a combination of Lie group theory with the cohomology theory of transformation groups - in the setting of geometric weight system $[\mathbf{4}, \mathbf{5}$, 8]. The geometric weight system of $X, \Omega(X)$, is a Weyl group invariant multiset in the weight lattice of the maximal torus $T$ of $G$-it is a generalization of the weight system of linear $G$-actions in the setting of cohomology theory (cf. §3). Conversely, such a $W$-invariant multiset of weights, $\Omega$, is called a $G$-weight pattern. We shall therefore be concerned with the following two problems which naturally arise.

Problem 1. Let $\Omega$ be a $G$-weight pattern (say, of a certain kind). If $\Omega$ is not of linear type (i.e. $\Omega \neq \Omega(\Phi)$ for any representation $\Phi$ ), how does one find sufficient conditions for $\Omega$ to be realizable as $\Omega(X)$ for some $G$-space $X$ ?

Received by the editors February 3, 1986.

1980 Mathematics Subject Classification (1985 Revision). Primary 57S15; Secondary 20GXX.

Research partially supported by the Norwegian Research Council for Science and Humanities (NAVF). 
PROBLEM 2. If $\Omega(X)$ is reasonably simple, how much of the orbit structure is "controlled" by $\Omega(X)$ ?

We mention that $\Omega(X)$ is, indeed, known to be closely related to the orbit structure data of $X$, and the complexity of the latter is somehow faithfully reflected by the complexity of $\Omega(X)$ (cf. [8]). Therefore, if the orbit structure of $X$ is rather simple, then its weight pattern must also be quite simple. A natural simplicity condition on $\Omega$ is given as follows. Assume $G=G(n)=O(n), U(n)$ or $\operatorname{Sp}(n)$ is a classical group of rank $>2$. A weight pattern $\Omega$ is called 2-parametric if each $\omega \in \Omega$ has an expression $\omega=\left(a \theta_{i}+b \theta_{j}\right)$ (in standard coordinates). In this paper we shall consider $G$-manifolds of acyclic or spherical type, having such weight systems, with the additional assumption that $\Omega(X)$ contains only one $W$-orbit of purely 2-parametric type. Real representations with such weight patterns are listed in Table I below (\#\# 1-9).

Let $V(\Phi)=\mathbf{R}^{N+1}$ (resp. $S^{N}$ ) be the representation space (resp. its restriction to the unit sphere) of the representation $\Phi$. As a notion of linear modeling, we say that a $G$-manifold $X \sim \mathbf{R}^{N+1}$ or $S^{N}$ is orthogonally modeled after a linear $G$-space $V(\Phi)$ if

$$
\begin{cases}\text { (i) } & \Omega(X)=\Omega(V(\Phi))(=\Omega(\Phi)) \\ \text { (ii) } \quad & X \text { and } V(\Phi) \text { have the same orbit types, i.e. } \\ & O(X)=O(V(\Phi)) ; \\ \text { (iii) } & \text { the corresponding slice representations are } \\ & \text { equivalent. }\end{cases}
$$

THEOREM A. Let $G$ be a classical compact Lie group acting differentiably on $X \sim_{\mathbf{Z}} \mathbf{R}^{N+1}$ or $S^{N}$, and assume the rational geometric weight system $($ cf. $\S 3(A))$, $\Omega_{0}^{\prime}(X)$, is of 2-parametric type and, moreover, there is only one 2-parametric $W$-orbit of weight pairs $( \pm \omega)$. Then there exists a 2-parametric real representation $\tilde{\Phi}=\Phi+l \cdot 1$ so that

$$
\Omega(X)=\Omega(\tilde{\Phi}), \quad \Omega_{p}(X)=\Omega_{p}(\tilde{\Phi}), \quad \forall p
$$

that is, $X$ has (integral) geometric weight system, as well as $\mathbf{Z}_{p}$-weight systems identical to those of $\tilde{\Phi}$. Moreover, $\Phi$ is listed in Table I.

THEOREM B. Let $G, X$ be as above, and assume $\Omega(X)=\Omega(\tilde{\Phi})$, where $\tilde{\Phi}=$ $\Phi+l \cdot 1$ and $\Phi$ is one of the representations listed in Table I. Then $X$ is orthogonally modeled on $V(\tilde{\Phi})=\mathbf{R}^{N+1}$ (resp. $S^{N}$ ), in particular, $X$ and $V(\tilde{\Phi})$ have the same orbit types. Moreover, with the possible exception of model \#2 or 3 in Table $\mathrm{I}$, the fixed point sets of any isotropy group $H$ in $X$ and $V(\tilde{\Phi})$ have the same cohomology (mod suitable coefficients $L=\mathbf{Z}$ or $\mathbf{Z}_{2}$ ), namely

$$
F(H, X) \sim_{L} F(H, V(\tilde{\Phi})), \quad \forall H \in O(X)=\mathcal{O}(V(\tilde{\Phi})) .
$$

THEOREM C. Let $G=G(m) \times G(n)$ be a product of classical groups (of the same type), and $X \sim \mathbf{Z} \mathbf{R}^{N+1}$ or $S^{N}$ a $G$-manifold. Suppose $\Omega_{0}^{\prime}(X)=\Omega_{0}^{\prime}(\Phi)$, $\Phi=\psi_{m} \otimes \psi_{n}+k \psi_{m}+k^{\prime} \psi_{n}$, that is, the nonzero weight system of $X$ coincides, 


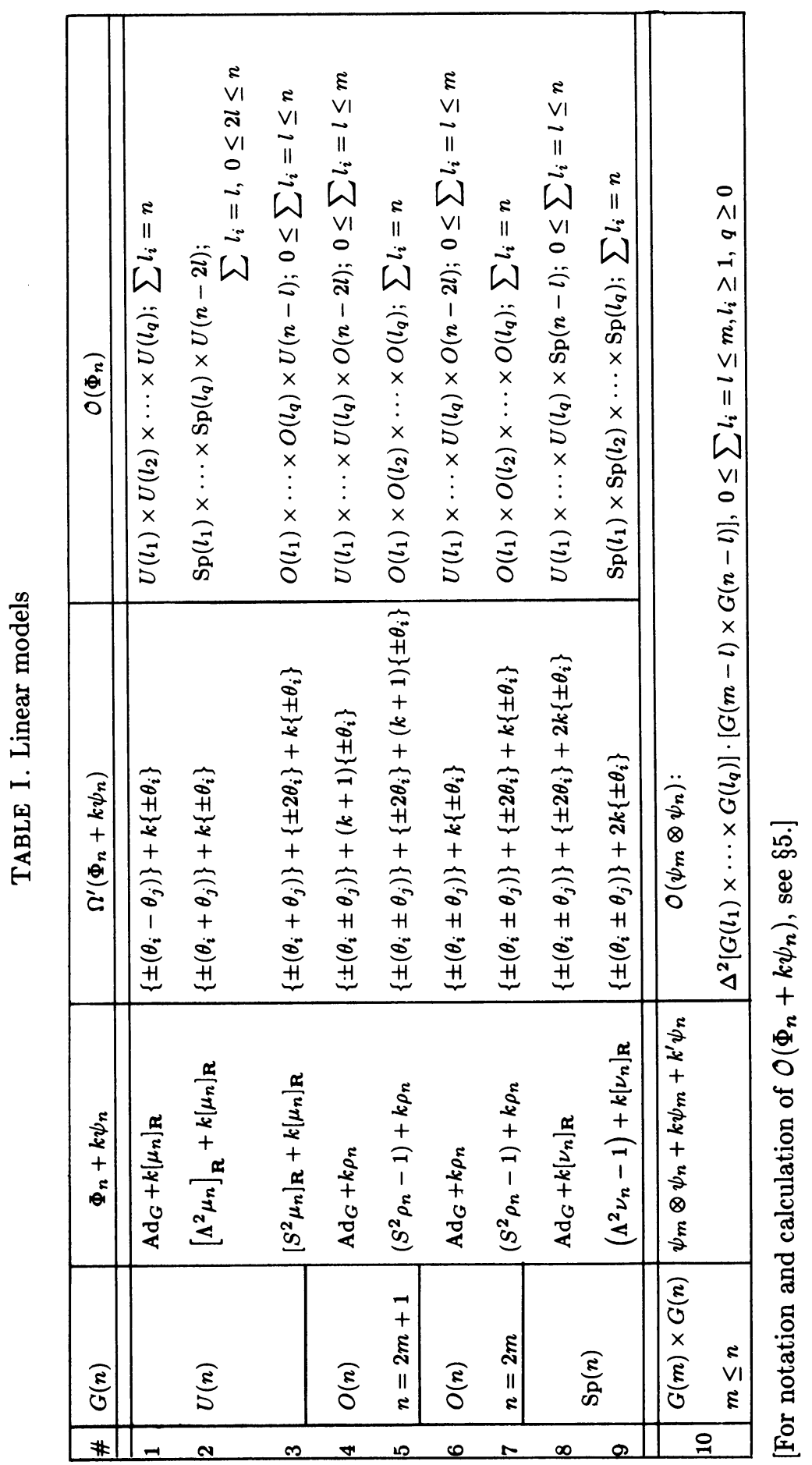


rationally at least, with that of $\Phi$ (cf. Table I). Then

(i) $\Omega(X)=\Omega(\tilde{\Phi}), \Omega_{p}(X)=\Omega_{p}(\tilde{\Phi}), \tilde{\Phi}=\Phi+l \cdot 1$ for some $l \geq 0$.

(ii) $X$ is orthogonally modeled on $V(\tilde{\Phi})=\mathbf{R}^{N+1}$ or $S^{N}$. Moreover, $F(H, X) \sim_{L}$ $F(H, V(\tilde{\Phi})), \forall H \in O(X)=O(V(\tilde{\Phi}))$, where $L=\mathbf{Z}$, or possibly $\mathbf{Z}_{2}$ in the orthogonal group case.

The basic idea of the proofs is to analyze the "interaction" between roots and weights. Weight patterns in the root lattice of $G$ are somehow more tightly connected with the root system, consequently the Weyl group $W$ exerts more control on the geometric behavior of $(G, X)$. This control is very rigid for the weight patterns in Table I. [For model \#2, 3 or 10, the appropriate "Weyl group" is not $W$, but these cases also fit into the scheme of our approach.]

We start in $\S 2$ by recalling some basic facts about $W$ and its role in the understanding of the orbit structure of the adjoint representation $\operatorname{Ad}_{G}$. For each choice of two roots, $\alpha, \beta$, there is associated a rank 2 group; these groups provide the typical linkage between the roots of $G$. Therefore, actions of rank 2 groups naturally constitute a system of pertinent substructures which are useful in analyzing $G$-actions of higher rank. In particular, Coxeter group techniques (i.e., reflection groups) are central in the proofs of the above theorems.

Our special study of rank 2 transformation groups (§4) will also be useful for further investigation of Problem 1, since consistency relations on rank 2 weight patterns also induce conditions on a general weight pattern via restrictions to different rank 2 "sections". Finally, we mention that a general study of minimal cohomogeneity, $\delta(G)$, as well as minimal complexity of $G$-weight pattern, for acyclic $G$-manifolds without fixed point is initiated in [7]. Based on [7] we now have, for example, $16 \leq \delta(S O(5)) \leq 19, \delta(S U(3)) \geq 24$, cf. $\S 4(\mathrm{E})$.

\section{2. $W$ and its induced actions.}

(A) $\mathrm{Ad}_{G}$ and subgroup structures. In the classical theory of compact connected Lie groups, the adjoint representation of $G, \operatorname{Ad}_{G}$, on its Lie algebra $\mathfrak{g}$ is a structural invariant of $G$ which is obtained via the partial linearization of the noncommutativity of $G$. The orbit structure of $\operatorname{Ad}_{G}$ can be neatly described in terms of the maximal torus theorem of É. Cartan and of the Weyl group $W=N(T) / T$, as follows. From the viewpoint of transformation groups, the Cartan algebra $\tau$ is recovered as the fixed point set of $T$ via $\operatorname{Ad}_{G}$, namely $T=F(T, \mathfrak{g})$, and one is led to consider the induced action of $W$ on $\tau$-let us call this the Cartan-Weyl representation, $(W, \tau)$. The orbit structure of $\operatorname{Ad}_{G}$ is "encoded" into this simpler action; the essential fact is that the principal isotropy type of $\operatorname{Ad}_{G}$ is $(T)$, and every $G$-orbit cuts $\tau$ (orthogonally) in points which constitute a $W$-orbit in $\tau$, in short

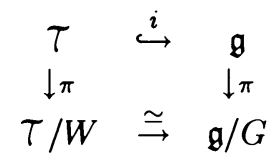

More generally, the same type of "reduction" also holds for the isotropy representation of a compact symmetric space $G / K$.

In the above situation $W$ acts effectively on $\tau$ as a group generated by reflections, and there is a 1-1 correspondence between isotropy types $\left(W_{x}\right)$ and $\left(G_{x}\right)$ such that $W_{x}=W_{G_{x}}$ is the Weyl group of $G_{x}=G_{x}^{0}, x \in \tau$. $W=W_{G}$, regarded as a linear 
group on $\tau$, contains a reflection $\rho_{\alpha}$ for each pair $( \pm \alpha)$ of roots. For a fixed system of simple roots $\left\{\alpha_{1}, \ldots, \alpha_{r}\right\} \subset \Delta(G)$ one obtains the corresponding simple system $\left\{\rho_{\alpha_{1}}, \ldots, \rho_{\alpha_{r}}\right\}$ of generators of $W$ (as Coxeter group). Any subgroup $K \subset W$ is contained in a unique smallest parabolic subgroup $\tilde{K}$ of $W$; parabolic means that $\tilde{K}$ is conjugate to a group generated by a subset of $\left\{\rho_{\alpha_{1}}, \ldots, \rho_{\alpha_{r}}\right\}$, in particular $\tilde{K}$ is a reflection subgroup.

Recall also the fundamental role of rank 2 groups, both in Coxeter group theory and in Lie theory, cf. [2] for standard properties. Later we shall, however, elaborate some of these facts in the setting of the Cartan-Weyl action associated with a $G$ manifold, cf. (B).

Let $\alpha, \beta, \ldots, \in \Delta(G)$ be roots and define subtori of $T$

$$
T \supset S_{\alpha} \supset S_{\alpha \beta} \supset S_{\alpha \beta \gamma} \supset \cdots, \quad S_{\alpha}=\alpha^{\perp}, \quad S_{\alpha \beta}=\left(S_{\alpha} \cap S_{\beta}\right)^{0}, \quad \text { etc. }
$$

Define corresponding subgroups of $G$ :

$$
\left\{\begin{array}{l}
G_{\alpha}^{\prime}=Z_{G}\left(S_{\alpha}\right), \quad G_{\alpha \beta}^{\prime}=\left\langle G_{\alpha}, G_{\beta}\right\rangle, \quad \text { etc. } \\
G_{\alpha}=G_{\alpha}^{\prime}, \quad G_{\alpha \beta}=Z_{G}\left(S_{\alpha \beta}\right), \quad \text { etc. }
\end{array}\right.
$$

where $Z_{G}(S)$ is the centralizer of the torus $S$. Note that the inclusion $G_{\alpha \beta \ldots}^{\prime} \subset$ $G_{\alpha \beta \ldots}$ may be strict, e.g., in $\operatorname{Sp}(n)$ let $\alpha=2 \theta_{1}, \beta=2 \theta_{2}$, so that $G_{\alpha \beta}^{\prime}=\left\langle T, \operatorname{Sp}(1)^{2}\right\rangle$ and $G_{\alpha \beta}=\langle T, \operatorname{Sp}(2)\rangle$.

The family of maximal rank connected subgroups of $G$ (up to conjugation) is precisely

$$
\left\{T, G_{\alpha}, \ldots, G_{\alpha \beta}^{\prime}, \ldots, G_{\alpha \beta \gamma}^{\prime}, \ldots, G\right\} .
$$

Each of these groups is uniquely determined by its Weyl group, as a subgroup of $W$. More generally, for any maximal rank group $H$ one can define its Weyl group

$$
W_{H}=[N(T) \cap H] / T \subset W, \quad T \subset H \subset G .
$$

LEMMA 2.1. $W_{H^{\circ}} \subset W_{H}$ is a normal subgroup, and is generated by reflections. $H$ is uniquely determined by the pair $\left(W_{H}, W_{H^{0}}\right)$, and in particular $W_{H} / W_{H^{0}}=$ $H / H^{0}$.

Proof. Clearly $H=\left\langle N(T) \cap H, H^{0}\right\rangle=\left\langle W_{H}, H^{0}\right\rangle$, and also

$$
H^{0}=\left\langle G_{\alpha_{1}}, G_{\alpha_{2}}, \ldots, G_{\alpha_{k}}\right\rangle
$$

where $\alpha_{i} \in\left\{\alpha \in \Delta(G) ; \rho_{\alpha} \in W_{H^{0}}\right\}$. The statement of the lemma now follows easily.

Not all reflection subgroups $W^{\prime} \subset W$ are of type $W_{H}, H$ connected. It is a standard result that isotropy groups of $\operatorname{Ad}_{G}$ are the centralizers of tori $S$, in fact, it suffices to consider only those $S$ of type (3). In short, we have

$$
\begin{array}{lc}
O\left(\operatorname{Ad}_{G}\right)=\left\{Z_{G}(S) ; S \subset T \text { of type }(3)\right\}= & \left\{G_{\alpha \beta \ldots}\right\} \\
\uparrow(6) & \cap ! \\
O(W, \tau)=\left\{\text { parabolic } W^{\prime} \subset W\right\} & \left\{G_{\alpha \beta \ldots}^{\prime}\right\}
\end{array}
$$

Let $G=G(n)=S O(n), S U(n)$ or $\mathrm{Sp}(n)$. The collection $O\left(\operatorname{Ad}_{G}\right)$ can be read off from Table I (by restricting to $S O(n)$ and $S U(n)$ ). But the collection $\left\{G_{\alpha \beta \ldots}^{\prime}\right\},(5)$, is larger (unless $G=S U(n)$ ). A more precise description, as well as generalization, of these subgroups are as follows. 
THEOREM 2.2. Let $G(n)$ be classical, as above, and let $H \subset G(n)$ be a closed connected subgroup of rank $>1$ having the same maximal torus as some $G(m) \subset$ $G(n)$ (standard imbedding). Then $H$ is given by

(i) $G=S U(n): H=S\left[U\left(l_{1}\right) \times U\left(l_{2}\right) \times \cdots \times U\left(l_{q}\right)\right] ; \sum l_{i}=m$.

(ii) $\overline{G=\mathrm{Sp}(n)}: H=\mathrm{Sp}\left(k_{1}\right) \times \cdots \times \mathrm{Sp}\left(k_{p}\right) \times U\left(l_{1}\right) \times \cdots \times U\left(l_{q}\right) ; \sum k_{i}+\sum l_{i}=m$.

(iii) $G=S O(n): H=S O\left(k_{1}\right) \times \cdots \times S O\left(k_{p}\right) \times U\left(l_{1}\right) \times \cdots \times U\left(l_{q}\right) ; \sum k_{i}+2 \sum l_{i} \leq$ $m$.

[Note! For $2 \sum l_{i}=n, l_{i}$ even, there is also $H^{\prime}=\sigma H$, where $\sigma$ is the outer automorphism induced by $O(n), c f .5 .1$.]

PROOF. This is a calculation involving root (and weight) spaces in $\mathfrak{g}$, bracket relations and representation theory. Since the case $m=n$ is well known, the cases (i) and (ii) are slightly simpler since we need only show $H \subset G(m)$. We shall illustrate the main idea of the proof by choosing $G=S U(n)$.

Let $S=S U(m) \cap T$ be a maximal torus of $H$, and suppose $H \not \subset S U(m), m \geq 3$. Since

$$
\Delta(G) \mid S=\Delta(S U(m))+(n-m) \Omega\left(\left[\mu_{m}\right]_{\mathbf{R}}\right) \quad(\bmod \text { trivial }),
$$

$\Delta(H)$ contains some $\theta_{1} \in \Omega\left(\left[\mu_{m}\right]_{\mathbf{R}}\right)$. The corresponding root space $\mathcal{N}_{1} \cong \mathbf{R}^{2}$ lies in $\mathfrak{g}_{1, m+1}+\cdots+\mathfrak{g}_{1, n}$, where $\mathfrak{g}_{1, i}$ is the root space of $\pm\left(\theta_{1}-\theta_{i}\right)$ in $\mathfrak{g}$. Choose a basis $v_{1}, v_{2}$ of $\mathcal{N}_{1}$ so that $\left[v_{1}, v_{2}\right]=t \neq 0$ lies in the (diagonal) Lie algebra of $S$. Since $v_{i}=\left(a_{1, m+1}^{(i)}+a_{1, m+2}^{(i)}+\cdots\right) \in \sum_{j>m} \mathfrak{g}_{1, j}$, calculations will show that $t$ must contain off-diagonal entries $\neq 0$, which is a contradiction.

(B) The Cartan-Weyl action of a $G$-manifold. If $X$ is a $G$-manifold, and $X^{T}=$ $F(T, X)$ is the fixed point set of the maximal torus $T$, then there is an induced action of $W$ on $X^{T}$, called the Cartan-Weyl action. The quotient of $W$ acting effectively on $X^{T}$ is denoted by $\bar{W}$ :

$$
1 \rightarrow W_{0} \rightarrow W \stackrel{\pi}{\rightarrow} \bar{W} \rightarrow 1,
$$

and a basic problem will be to calculate $\bar{W}$ (or $W_{0}$ ), say, in terms of the weight system $\Omega(X)$ (defined in §3). For a given $G$-action $\varphi$ the $\bar{W}$-action is sometimes written $\bar{\varphi}$.

$(\bar{W}, \bar{\varphi})$ can be analyzed by considering certain Cartan-Weyl actions $\left(\bar{W}_{*}, \bar{\varphi}_{*}\right)$ of $H=G_{*}(*=$ string of roots $=\alpha \beta \ldots)$. Since the weight lattice of $T / S$ can be regarded as a sublattice of the weight lattice of $T$, the root system of the semisimple group $\bar{G}_{*}^{\prime}=G_{*}^{\prime} / S_{*}$ can be identified with $\Delta\left(G_{*}^{\prime}\right) \subset \Delta(G)$. One verifies that

$$
\left.\begin{array}{l}
\Delta\left(G_{*}^{\prime}\right) \\
\Delta\left(G_{*}\right)
\end{array}\right\}=\Delta(G) \cap\left\{\begin{array}{l}
\mathbf{Z} \cdot \alpha+\mathbf{Z} \cdot \beta+\cdots, \\
\mathbf{Q} \cdot \alpha+\mathbf{Q} \cdot \beta+\cdots,
\end{array}\right.
$$

where $\mathbf{Z} \cdot \alpha+\mathbf{Z} \cdot \beta+\cdots$ is the root lattice of $G_{*}^{\prime} \cdots$. Now, (9) gives the (nonzero) weights of adjoint representations on $\mathfrak{g}_{*}^{\prime}, \mathfrak{g}_{*}$. Hence, if $Z(H)$ denotes the center of $H$, then

$$
\begin{array}{r}
F\left(S_{*}, \mathfrak{g}\right)=F\left(Z\left(G_{*}\right), \mathfrak{g}\right)=\mathfrak{g}_{*} \\
\quad F\left(Z\left(G_{*}^{\prime}\right), \mathfrak{g}\right)=\mathfrak{g}_{*}^{\prime} .
\end{array}
$$


Similarly, we can define subactions of $(G, \varphi, X)$

$$
\left\{\begin{array}{c}
{ }^{\prime} \varphi_{*}=\text { action of } G_{*} \text { on }{ }^{\prime} X_{*}=F\left(Z\left(G_{*}\right), X\right), \\
\cap \\
\varphi_{*}=\text { action of } G_{*} \text { on } X_{*}=F\left(S_{*}, X\right), \\
\cup \\
\varphi_{*}^{\prime}=\text { action of } G_{*}^{\prime} \text { on } X_{*}^{\prime}=F\left(Z\left(G_{*}^{\prime}\right), X\right) .
\end{array}\right.
$$

Assuming these actions have well defined (geometric) weight system, the analog of (9) will be

$$
\begin{aligned}
&\left\{\begin{array}{l}
\Omega^{\prime}\left({ }^{\prime} X_{*}\right) \\
\Omega^{\prime}\left(X_{*}\right) \\
\Omega^{\prime}\left(X_{*}^{\prime}\right)
\end{array}\right\}=\Omega^{\prime}(X) \cap\left\{\begin{array}{l}
R_{G} . \\
\mathbf{Q} \cdot \alpha+\mathbf{Q} \cdot \beta+\cdots \\
\mathbf{Z} \cdot \alpha+\mathbf{Z} \cdot \beta+\cdots
\end{array}\right. \\
&\left({ }^{*}=\alpha \beta \ldots\right)
\end{aligned}
$$

Here $R_{G_{*}}=$ (root lattice of $G_{*}$ ) is generated by $\Delta(G) \cap[\mathbf{Q} \cdot \alpha+\mathbf{Q} \cdot \beta+\cdots]$. Let $\left(\bar{W}_{*}, \bar{\varphi}_{*}\right)$ be the Cartan-Weyl action of some action in (10). Then $\bar{W}_{*}$ can be naturally identified with a subgroup of $\bar{W}$,

$$
\begin{array}{lll}
W & \stackrel{\pi}{\rightarrow} & \bar{W} \rightarrow 1 \\
\uparrow_{i} & & \uparrow i \\
W_{*} & \rightarrow & \bar{W}_{*} \rightarrow 1
\end{array}
$$

(C) Topological reflection groups. In many cases to be considered in the sequel, $\bar{W}$ acts on $X^{T}$ as a group generated by (topological) reflections. We refer to [3, III] or [11] for the "well-behaved" (i.e., similar to the linear case) properties of such groups. We shall also need the following result.

THEOREM 2.3. Let $W \stackrel{\pi}{\rightarrow} \bar{W}$ be a surjective homomorphism from the finite Coxeter group $W$ to a (topological) reflection group $\bar{W}$ on a (topological) space $X$. Assume $\pi$ maps reflections to reflections. Then $\pi$ is an isomorphism if and only if $\pi$ is injective on each parabolic subgroup of rank 2 .

PrOOF. We know $\bar{W}$ is a Coxeter group (cf. [11, p. 434]). If $\rho_{1}, \rho_{2}$ are two reflections in $W$, then there is a parabolic subgroup $W_{2}$ of rank 2 containing $\left\{\rho_{1}, \rho_{2}\right\}$. Therefore, the theorem is a direct consequence of the proposition below.

PROPOSITION 2.4. Let $W \stackrel{\pi}{\rightarrow} \bar{W}$ be a surjective homomorphism between finite Coxeter groups. Let

$$
R=\{\text { reflections in } W\}, \quad \bar{R}=\{\text { reflections in } \bar{W}\} \text {. }
$$

Suppose $\pi$ maps $R$ into $\bar{R}$ injectively. Then $\pi$ is an isomorphism, more precisely, $\mathrm{rk} W=\mathrm{rk} \bar{W}$ and a simple system of generators maps onto a simple system.

PROOF OF 2.4. Let $\hat{R}=\pi(R) \subset \bar{R}$, and observe that $\hat{R}$ satisfies

(i) $\hat{R}$ generates $\bar{W}$,

(ii) $x \hat{R} x^{-1}=\hat{R}, \forall x \in \bar{W}$.

From the general theory of (abstract) Coxeter groups it follows that (12) implies $\hat{R}=\bar{R}$, so $\# R=\# \bar{R}$. Using standard arguments in Coxeter group theory one proves next that $W$ and $\bar{W}$ also have the same rank. Now, it is easy to reduce to the case of irreducible Coxeter groups $W, \bar{W}$, and then it is a simple matter to check (e.g., by classification, comparison of order) that $W \simeq \bar{W}$. 


\section{Some basic tools.}

(A) The geometric weight system. Let $G$ be a compact connected Lie group, $T$ a maximal torus, and $X$ an acyclic $G$-manifold. Then by Smith theory $F(T, X)=X^{T}$ is also acyclic, and the local representation of $T$ at an arbitrary point $x \in X^{T}$ is well defined-its weight system is called the geometric weight system of $X$ (or the action $\varphi$ ), and is denoted by $\Omega(X)$ (or $\Omega(\varphi)$ ). The collection of nonzero weights $( \pm \omega)$ is denoted by $\Omega^{\prime}(X)[\mathbf{4}, \mathbf{5}, \mathbf{8}]$. Next, let $X \sim S^{N}$ be a cohomology sphere, and so $X^{T} \sim S^{r}, r \equiv N(\bmod 2), r \geq-1$. For $X^{T} \neq \varnothing$ we define the geometric weight system as above, except that the zero weight is given multiplicity $\left(\operatorname{dim} X^{T}+1\right)$. Finally, if $X \sim S^{\text {odd }}$ and $X^{T}=\varnothing$, then the concept of weight system is still defined if we do not distinguish between proportional weights (cf. splitting principle, $[\mathbf{4}, \mathbf{5}]$ ). This gives the rational geometric weight system, $\Omega_{0}^{\prime}(X)$, consisting of those corank one subtori $H$ with $m(H)=\left[\operatorname{dim} X^{H}-\operatorname{dim} X^{T}\right]>0$, called the multiplicity of $H$. One may identify $H$ with the unique pair $( \pm \bar{\omega})$ of primitive weights in the weight lattice of $T$ such that $\bar{\omega}^{\perp}=H$. We mention, however, that the integral weight system may sometimes be well defined even if $X^{T}=\varnothing$, namely, $\Omega_{0}^{\prime}(X)$ is derivable from a collection of weights with multiplicities, $\Omega^{\prime}(X)$, whose restrictions $\Omega^{\prime}(X) \mid H$ give the weight systems of the local representations of all $H \in \Omega_{0}^{\prime}(X)$. Note that the total multiplicity of $\Omega_{0}(X)$ or $\Omega(X)$ equals $\operatorname{dim} X$ (resp. $\left.\operatorname{dim} X+1\right)$ if $X$ is acyclic (resp. spherical). Since $\Omega^{\prime}(X), \Omega_{0}^{\prime}(X)$ are invariant under the natural action of the Weyl group, we also write weight systems additively as sums of $W$-orbits. Let $\varphi_{x}$ be the slice representation of $G_{x}$ and $S$ a maximal torus of $G_{x}$. Since the isotropy representation of $G_{x}$ in the tangent space of $G(x)$ at $x$ is $\tau_{x}=\operatorname{Ad}_{G} \mid G_{x}-\operatorname{Ad}_{G_{x}}$, one has

$$
\Omega^{\prime}(X \mid S)=\Omega^{\prime}\left(\tau_{x}\right)+\Omega^{\prime}\left(\varphi_{x}\right)=\Delta(G) \mid S-\Delta\left(G_{x}^{0}\right)+\Omega^{\prime}\left(\varphi_{x}\right) .
$$

Now, let $p$ be a prime number and $T_{p} \cong \mathbf{Z}_{p}^{q}$ a (maximal) $\mathbf{Z}_{p}$-torus of $G$. Then

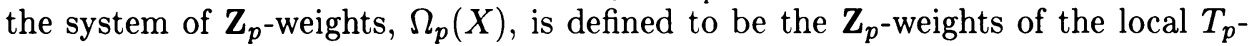
representation at a point $x \in \mathbf{F}\left(T_{p}, X\right)$ (or via the splitting principle if $F\left(T_{p}, X\right)=$ $\varnothing)$. Technically, one can work with $\mathbf{Z}_{p}$-weight systems in a way very similar to the "usual" case, in particular, the $p$-version of $(3.1)$ holds. We refer to $[\mathbf{4}, 9]$ for definition as well as applications of $\mathbf{Z}_{p}$-weights to the calculation of orbit types. $\Omega_{p}(X)$ is invariant under the action of the $\mathbf{Z}_{p}$-Weyl group, $W_{p}=N\left(T_{p}\right) / T_{p}$; the most important case will be $p=2$.

NOTE. In general, the $G$-spaces involved will be acyclic or spherical $(\bmod L)$. $\Omega_{p}(X)$ will be defined (by Smith theory) when $L=\mathbf{Z}_{p}$; in the case of $\Omega(X)$ or $\Omega_{0}(X)$ it suffices to have $L=\mathbf{Q}$.

(B) Slices and isotropy groups. Here we shall combine the slice theorem with the structure of compact Lie groups to establish the following useful theorem, and later we briefly discuss how isotropy groups may be calculated.

THEOREM 3.2. Let $K \subset G$ be compact Lie groups ( $K$ possibly finite), and $Y$ a connected smooth $G$-manifold. Let $S_{x}$ denote a slice at $x$, and define subsets of $Y^{K}=F(K, Y)$

$$
Y_{e}^{K}=\left\{x ; \exists y \in S_{x}, G_{y}=K\right\}, \quad Y_{0}^{K}=\left\{x ; \exists y \in S_{x}, G_{y} \supset K, G_{y}^{0}=K^{0}\right\} ;
$$

and if $K$ is a torus $(p=0)$ or $\mathbf{Z}_{p}$-torus ( $p$ prime)

$$
Y_{t}^{K}=\left\{x ; \exists y \in S_{x}, K \subset G_{y} \text { and is maximal }\right\} .
$$

Then $Y_{e}^{K}, Y_{0}^{K}, Y_{t}^{K}$ are closed and open subsets of $Y^{K}$. 
COROLlaRY 3.3 Assume $F(K)$ connected, $K \subset G_{x} \subset G_{y}, K^{0}=G_{x}^{0}$. Then for some $z \in S_{y} G_{z} \supset K$ and $G_{z}^{0}=K^{0}$.

COROLlaRY 3.4. Let $K=S=$ torus, $F(S)$ connected. Suppose $S \subset G_{x}$ is a maximal torus, and $S \subset G_{y}$. Then for some $z \in S_{y} S$ is a maximal torus of $G_{z}$.

PROOF OF THEOREM 3.2. Let $Y_{*}^{K}$ denote one of the three types of subspaces of $Y^{K}$, and note first that $Y_{*}^{K}$ is $N(K)$-invariant. We shall make use of the following lemma, whose proof involves standard slice arguments and properties of subgroups of $G$.

LEMma. Let $x \in Y^{K}$. Then there is a nbd $U$ of $e \in G$ such that the nbd $\tilde{U}=U \cdot S_{x} \subset Y$ of $x$ satisfies

$$
\tilde{U} \cap Y^{K}=\tilde{U} \cap\left[Z_{G}(K)^{0} \cdot S_{x}^{K}\right],
$$

where $Z_{G}(K)^{0}$ is the connected centralizer of $K$ in $G$. Moreover,

$$
\tilde{U} \cap Y_{*}^{K}=\tilde{U} \cap\left[Z_{G}(K)^{0} \cdot\left(S_{x}\right)_{*}^{K}\right],
$$

where $\left(S_{x}\right)_{*}^{K}$ refers to the action of $G_{x}$ on $S_{x}$.

Now, we sketch the proof of 3.2 , as follows. By induction on $\operatorname{dim} Y$, we assume $\operatorname{dim} Y>0$ and divide into two cases.

(i) Linear case. $G$ acts linearly on $V=\mathbf{R}^{m}, m \geq 1$. We claim that $V_{*}^{K}=V^{K}$ or $V_{*}^{K}=\varnothing$; first we check the case $m=1$, and then for $m \geq 2$ we may pass to the unit sphere $S(V)$ and use induction on dimension.

(ii) General case. $\operatorname{dim} Y=m \geq 1$. Assume the theorem true for all connected $G$-manifolds of $\operatorname{dim}<m$ and also if $Y$ is linear. Let $x \in Y^{K}, \tilde{U}=U \cdot \mathcal{S}_{x}$ as in the lemma. By hypothesis, for the linear action $\left(G_{x}, S_{x}\right)$ we have either (a) $\left(S_{x}\right)_{*}^{K}=S_{x}^{K}$, or (b) $\left(S_{x}\right)_{*}^{K}=\varnothing$. In case (a) the lemma implies $\tilde{U} \cap Y_{*}^{K}=\tilde{U} \cap Y^{K}$, and in case (b) $\tilde{U} \cap Y_{*}^{K}=\varnothing$. Hence, $Y_{*}^{K}$ is closed and open in $Y^{K}$.

The torus orbit types of the $G$-manifold $X$ is, by definition, the collection $O^{t}(X)$ of all conjugacy classes $(S)$, where $S$ is a maximal torus of some isotropy group $G_{x}$. We may assume $S=\left(T \cap G_{x}\right)^{0}$, and then $S$ can be expressed as

$$
S=\left(\omega_{1}^{\perp} \cap \omega_{2}^{\perp} \cap \cdots \cap \omega_{k}^{\perp}\right)^{0}, \quad \omega_{i} \in \Omega_{0}(X) .
$$

However, not all choices of $\omega_{i}$ lead to some $S \in \mathcal{O}^{t}(X)$. There exists a (purely combinatorial) algorithm which calculates $O^{t}(X)$ from $\Omega_{0}^{\prime}(X)$ and $\Delta(G)$, cf. $[\mathbf{5}, \mathbf{8}]$. By this algorithm, the "if" part of Lemma 3.5 is trivial. In the case considered in 3.5 , however, we shall give a complete proof, using induction on Lie group structure.

LEMMA 3.5. Let $G$ be compact connected, $X \sim$ pt or $S^{N}, \Omega_{0}^{\prime}(X)=\Delta(G)+\Gamma$. Then $S \subset T$ belongs to $O^{t}(X)$ if and only if $S$ can be expressed as in (13) with all $\omega_{i} \in \Gamma$.

ProOF. Assume the lemma holds for all proper connected subgroups of $G$. We may assume $G \neq T, \Delta(G) \not \subset \Gamma$ (by the algorithm), and then $F(G) \neq F(T) \neq \varnothing$ (by $\S 3(\mathrm{C}))$. So there is some $G_{y}, T \subset G_{y} \subsetneq G$, and assume $S \subset T$ is maximal in 
some $G_{x}$. By Corollary $3.4 S$ is also maximal in some $G_{z}$ in the slice representation $\varphi_{y}$ of $G_{y}$. By (3.1)

$$
\begin{aligned}
\Omega_{0}^{\prime}(X) & =\Delta(G)+\Gamma=\Delta(G)-\Delta\left(G_{y}^{0}\right)+\Omega_{0}^{\prime}\left(\varphi_{y}\right) \\
& \Rightarrow \Omega_{0}^{\prime}\left(\varphi_{y}\right)=\Delta\left(G_{y}^{0}\right)+\Gamma .
\end{aligned}
$$

Hence, the lemma follows by induction.

The calculation of $O(X)$, the collection of isotropy types, generally consists of three steps: $O^{t}(X) \rightarrow O^{0}(X) \rightarrow O(X)$, as follows. First, the maximal tori of isotropy groups are determined, as explained above. The collection $O^{0}(X)$ consists of connected isotropy types $\left(G_{x}^{0}\right)$. If we know a maximal torus $S \subset G_{x}^{0}$, then the structure of $G_{x}^{0}$ sometimes follows from equation (3.1). In the final stage, $\mathbf{Z}_{p}$-weight systems $\Omega_{p}^{\prime}(X)$ may be used to "control" the $p$-torsion of $G_{x} / G_{x}^{0}$; here the $p$-version of (3.1) and Theorem 3.2 can be useful (cf. §5).

(C) Some applications of $S O(3)$-actions. Let $S O(3)$ act on $X \sim \mathbf{z}_{2} S^{N}, N \neq 3$, with $F(S O(2))=\varnothing$. Then by Smith theory $N$ is odd, and it is not too difficult to show $N \geq 7$. The following theorem is, however, meaningful only when $\Omega^{\prime}(X)$ is well defined, so we shall assume $F(S O(2)) \neq \varnothing$ in the spherical case.

THEOREM 3.6. Let $X \sim \mathbf{z}_{2}$ pt or $S^{N}$ be an $S O(3)$-manifold, let $\alpha$ be the unit weight, and put $\delta_{1}=\sum d_{\mathrm{odd}}, \delta_{2}=\sum d_{\mathrm{even}}$, where

$$
\varnothing \neq \Omega^{\prime}(X)=d_{1}\{ \pm \alpha\}+d_{2}\{ \pm 2 \alpha\}+\cdots .
$$

Then (i) [codim of $F(O(2))$ in $F(S O(2))]=\delta_{1}-\delta_{2} \geq 0, d_{1} \geq 1$,

(ii) $\delta_{2}=0 \Rightarrow F(S O(3))=F(O(2)) \sim \mathbf{z}_{2}$ pt or $S^{N-3 \delta_{1}}$.

PROOF. In the acyclic case, or if $F(O(2)) \neq \varnothing$, this follows from [6, Lemma 2.1]. Assume therefore $X \sim S^{N}$ and $F(O(2))=S^{-1}$. Using (3.1) we find $d_{1} \geq 1$. Let $F(S O(2)) \sim S^{r}, N=r+2 \delta_{1}+2 \delta_{2}, F\left(\mathbf{Z}_{2}\right) \sim S^{r+2 \delta_{2}}$. Now, choose a suitably large dihedral 2-group $D_{2^{k}}$ in $S O(3)$ so that $F\left(\mathbf{Z}_{2^{k}}\right)=F(S O(2)), F\left(D_{2^{k}}\right)=S^{-1}$. Using [10, Theorem 4.6], $(N-r) / 2=\left(r+2 \delta_{2}-(-1)\right)$, or equivalently $r+1=\delta_{1}-\delta_{2}$. This proves (i).

Next, assume $\delta_{2}=0$. Since $F(O(2))=S^{-1}, F\left(\mathbf{Z}_{2}\right) \sim S^{r}$, we find $F\left(D_{2}\right)=$ $S^{-1}$, and Borel's formula (cf. [1, Chapter XIII]) applied to the action of $D_{2}$ gives $N+1=3[r+1]$, that is, $r-\delta_{1}=N-3 \delta_{1}=-1$ and (ii) follows.

We shall now apply the above theorem to get information about the Cartan-Weyl action $\left(\bar{W}, X^{T}\right)$ of a $G$-manifold $X \sim \mathbf{z}_{2}$ pt or $S^{N}$ with a given geometric weight system. Write the latter as

$$
\Omega^{\prime}(X)=\sum_{k \geq 1} \sum_{\alpha} d_{k}^{\alpha} \cdot\{ \pm k \alpha\}+\Gamma \quad\left(\alpha \in \Delta^{+}(G)\right),
$$

where $\Gamma$ contains no multiple $k \alpha, k \geq 1$, and put $\delta_{1}^{\alpha}=\sum d_{\text {odd }}^{\alpha}, \delta_{2}^{\alpha}=\sum d_{\text {even }}^{\alpha}$. Since the $S O(3)$-action $\left(\bar{G}_{\alpha}, X_{\alpha}^{\prime}, \varphi_{\alpha}^{\prime}\right)$, cf. (10), (11), has weight system $\Omega^{\prime}\left(\varphi_{\alpha}^{\prime}\right)=$ $\sum_{k} d_{k}^{\alpha}\{ \pm k \alpha\}$, the following is a consequence of 3.6.

Proposition 3.7. Let $X, G$ be as above. Then $\delta_{1}^{\alpha} \geq \delta_{2}^{\alpha}$ and, moreover,

(i) $\bar{\rho}_{\alpha}=1$ in $\bar{W}$ if and only if $\delta_{1}^{\alpha}=\delta_{2}^{\alpha}$, and hence $\bar{W}=1$ if and only if $\delta_{1}^{\alpha}=\delta_{2}^{\alpha}$, $\forall \alpha \in \Delta(G)$. 

$\forall \alpha$;

(ii) $\bar{W}$ is a group generated by (topological) reflections if and only if $\delta_{1}^{\alpha}-\delta_{2}^{\alpha} \leq 1$,

(iii) assume $\delta_{2}^{\alpha}=0, \forall \alpha$. Then

$$
F(H, X)=F\left(W_{H}, X^{T}\right), \quad \forall H \supset T, H=H^{0} .
$$

REMARK 3.8. In view of Lemma 2.1, consider the category of $G$-actions $\varphi$ on spaces $X$ satisfying property (14). Then $\forall x \in X^{T} G_{x}$ is uniquely determined by the group $W_{x} \subset W$ (i.e., independent of $\varphi$ ). In particular, $W_{x}=W_{G_{x}}$, cf. (6).

PROPOSITION 3.9. Let $G=S O(n)$ ( $n$ even), $S U(n)$ or $\operatorname{Sp}(n)$, and let $\psi_{n}$ be the standard representation of $G\left(\operatorname{dim}_{\mathbf{R}} \psi_{n}=n, 2 n, 4 n\right.$ resp. $)$. Assume $\Omega^{\prime}(X)=\Omega^{\prime}(\Phi)$, $\Phi=\operatorname{Ad}_{G}+k \psi_{n}, k \geq 0$. Then $W \simeq \bar{W}$ acts faithfully on $X^{T}$ as a group generated by reflections. Moreover, $X$ and $V(\Phi)$ have the same maximal rank isotropy types.

ProOF. Let $\alpha, \beta$ be simple roots. Then $G_{\alpha \beta}=G_{\alpha \beta}^{\prime}$, and it is easy to check that $\Omega^{\prime}\left(\psi_{n}\right) \cap[\mathbf{Z} \cdot \alpha+\mathbf{Z} \cdot \beta]=\varnothing$. Hence, by $(11), \Omega^{\prime}\left(X_{\alpha \beta}^{\prime}\right)=\Delta\left(G_{\alpha \beta}\right)$. From the results on rank 2 groups in $\S 4$ it now follows that $\bar{W}_{\alpha \beta} \simeq W_{\alpha \beta}$, and hence Theorem 2.3 applies to show $W \simeq \bar{W}$ (as Coxeter groups). Moreover, Remark 3.8 applies to both $X$ and $V(\Phi)$, and their Cartan-Weyl actions have the same isotropy groups $W_{x}$, namely the parabolic subgroups of $W$.

(D) On simple weight patterns related to $\Delta(G)$. Since $\operatorname{Ad}_{G}$ plays such a unique role in representation theory, in view of $\S 2$ and the above results it is natural to inquire what are the linear actions $\Psi$ "closest" to $\operatorname{Ad}_{G}$, say, in terms of the behavior of Weyl group, orbit structure etc. Recall that $\operatorname{Ad}_{G}$ (mod trivial copies) is uniquely characterized by the simple fact that the principal isotropy type is $(T)$. Moreover, $O\left(\operatorname{Ad}_{G}\right)$ can be computed effectively using the fact that (a) $W \simeq \bar{W}$ is a reflection group on $\tau$, and (b) property (14) holds. By focusing attention on (a) and (b), an appropriate notion of "closeness" may be chosen as follows.

First, let $G$ be simple, rk $G \geq 2$. The following two conditions on a real representation $\Psi$ of $G$ are equivalent (cf. 3.7):

(i) $\bar{W} \neq 1$, and is generated by reflections. Moreover, property (14) is satisfied;

$$
\left\{\begin{array}{l}
\text { (ii) } \Omega^{\prime}(\Psi) \text { contains roots, and these have multiplicity } 1 . \\
\text { Furthermore, } \Omega^{\prime}(\Psi) \text { contains no root multiple } k \alpha, k>1 .
\end{array}\right.
$$

NOTE. For the implication (i) $\Rightarrow$ (ii) one needs the fact that if $\Omega^{\prime}(\Psi)$ contains some $k \alpha, k \geq 3$, then $\alpha$ has multiplicity $>1$. One checks this by suitably reducing to the case rk $G=2$ as in $\S 2(\mathrm{~B})$.

DEFINITION. A faithful real representation ${ }^{1} \Psi$ of a semisimple compact connected Lie group $G / Z(G)$ is called adjoint similar if $\Omega^{\prime}(\Psi)$ satisfies condition (15).

Now, let $G$ be simple and $\Psi$ as above. Then $\Psi$ is irreducible, ${ }^{2}$ and besides the obvious candidates $\operatorname{Ad}_{G}$ or $\rho$, where $\Omega^{\prime}(\rho)=$ short roots $\}$, there turn out to be just the infinite family $\left(S^{2} \rho_{2 n}-1\right)$ of $S O(2 n) / \mathbf{Z}_{2}$ and $\Lambda^{4} \nu_{4}-\Lambda^{2} \nu_{4}$ of $\operatorname{Sp}(4) / \mathbf{Z}_{2}$. Hence, the possibilities of $\bar{W}$ are listed in Table II.

${ }^{1}$ Modulo trivial summands.

${ }^{2}$ See footnote 1 . 
Comments. (i) Let $G \sim G_{1} \times \cdots \times G_{l}$ be semisimple, $\Psi=\Psi_{1} \otimes \cdots \otimes \Psi_{l}$. Then $\Psi$ is adjoint-similar if and only if each factor is so, with $\bar{W}_{i}=S_{2}$ (i.e. $\operatorname{rk} \bar{W}_{i}=1$ ). A reducible $\Psi$ must be the outer direct sum of adjoint-similar summands $\Psi_{i}=\Psi \mid G_{i}$.

(ii) For $G=S O(8) / \mathbf{Z}_{2}$ there are actually two more $\Psi$, namely those mapped to $\left(S^{2} \rho_{8}-1\right)$ via outer automorphisms. Correspondingly, $W$ has three different quotients $\simeq S_{4}$.

(iii) Observe that Table II does not include all irreducible representations of simple groups of rank $\geq 2$ with $\bar{W} \neq 1$ acting as reflection group, e.g., the representation $\left(S^{2} \rho_{2 n+1}-1\right)$ of $S O(2 n+1)$ is missing. The latter fits, however, into the scheme of $\S 5$, cf. Table I.

(iv) Let $G \sim B_{n}, C_{n}, G_{2}$ or $F_{4}$, and consider actions $\varphi$ on spaces $X \sim \operatorname{pt}$ or $S^{N}$. Then the $G$-weight pattern

$$
\Omega^{\prime}=\{\text { long roots }\}+\Gamma, \quad \Gamma \neq \varnothing,
$$

where $\Gamma$ contains no short roots, cannot be realized as $\Omega^{\prime}(\varphi)$.

(v) For $G$-manifolds $X$ having the weight pattern of adjoint-similar representations $\Psi$, one can prove (in a uniform way) regularity theorems precisely similar to Theorem B of $\S 1$. In fact, since all cases of Table II have nontrivial principal isotropy groups, such theorems have been known for some time (cf. e.g., $[\mathbf{3}, \mathbf{1 2}]$ ).

TABLE II. Adjoint-similar representations of simple groups

\begin{tabular}{ccc}
\hline$G$ & $\Psi$ & $1 \rightarrow W_{0} \rightarrow W \rightarrow \bar{W} \rightarrow 1$ \\
\hline$G$ & Ad & $W_{0}=1$ \\
$S O(2 n+1)$ & $\rho_{2 n+1}$ & $W_{S O(2 n)} \rightarrow W \rightarrow S_{2}$ \\
$\mathrm{Sp}(n) / \mathbf{Z}_{2}$ & $\rho=\Lambda^{2} \nu_{n}-1$ & $W_{\mathrm{Sp}(1)^{n}} \rightarrow W \rightarrow S_{n}$ \\
$G_{2}$ & $\rho_{7}$ & $W_{S U(3)} \rightarrow D_{6} \rightarrow S_{2}$ \\
$F_{4}$ & $\rho_{26}$ & $W_{\mathrm{Spin}(8)} \rightarrow W \rightarrow S_{3}$ \\
$S O(2 n) / \mathbf{Z}_{2}$ & $S^{2} \rho_{2 n}-1$ & $\mathbf{Z}_{2}^{n-1} \rightarrow W \rightarrow S_{n}$ \\
$\mathrm{Sp}(4) / \mathbf{Z}_{2}$ & $\Lambda^{4} \nu_{4}-\Lambda^{2} \nu_{4}$ & $D_{2} \hat{\times} W_{\mathrm{Sp}(1)^{4}} \rightarrow W \rightarrow S_{3}$ \\
\hline
\end{tabular}

4. Analysis of weight pattern for groups of rank 2. In this section $G$ is a compact connected (semi-) simple group of rank 2. Recall that such groups and their induced actions (cf. $\S \S 1$ and 2) provide the linking substructure used to analyze actions of higher rank groups. For this reason the cases $G \sim S U(3)$ or $\operatorname{Sp}(2)$ are the most important ones; the special case $G=G_{2}$ is of "isolated" interest, just as it is in the Lie theory. Therefore, as far as the cases $G \sim S U(2) \times S U(2)$ or $G_{2}$ are concerned, we shall only state the analogous results stated and proved for $S U(3)$ and $\operatorname{Sp}(2)$.

In (A)-(D) we look closely at the relationship between $\left(\bar{W}, X^{T}\right)$ and the possible $G$-weight pattern $\Omega^{\prime}(X)$, leading to an explicit calculation of $\bar{W}$. This also imposes consistency relations on $\Omega^{\prime}(X)$ (cf. Problem 1, §1). An immediate application of this will be given in $(\mathrm{E})$. Certain types of regularity theorems are rather easily obtained for rank 2 groups-an example is given in $(\mathrm{F})$. 
For a given $G$-manifold $X$, split the weight system as

$$
\Omega(X)=\Omega\left(X^{\prime}\right)+\left[\Omega(X) \backslash R_{G}\right]
$$

i.e., $\Omega\left(X^{\prime}\right)=\Omega(X) \cap R_{G}$ consists of those weights lying in the root lattice $R_{G}$. As indicated by notation, $\Omega\left(X^{\prime}\right)$ is the weight system of the $G$-space $X^{\prime}=F(Z(G), X)$, where $Z(G)=1, \mathbf{Z}_{2}, \mathbf{Z}_{2} \times \mathbf{Z}_{2}$ or $\mathbf{Z}_{3}$ is the center of $G$. As for linear actions, $\Omega(X) \backslash R_{G}$ has no effect on the Cartan-Weyl action $\left(\bar{W}, X^{T}\right)$, i.e. the latter depends only on $X^{\prime}$ (cf. Remarks 4.2 below).

(A) $G=S U(3)$. The root lattice is the collection of weights

$$
R_{G}: \omega=\left(n \theta_{i}+m \theta_{j}\right) ; \quad n+m \equiv 0 \quad(\bmod 3),
$$

where $\left(\theta_{1}, \theta_{2}, \theta_{3}\right), \sum \theta_{i}=0$, refers to $T=\operatorname{diag}\left\langle e^{2 \pi i \theta_{1}}, e^{2 \pi i \theta_{2}}, e^{2 \pi i \theta_{3}}\right\rangle$. The weight lattice of $S U(3)$ consists of all $\omega=\left(n \theta_{i}+m \theta_{j}\right)$; we shall arrange them into three classes:

$$
\begin{cases}\underline{\text { type (a) }} & n \not \equiv 0, m \not \equiv 0, n-m \not \equiv 0(\bmod 3) \\ & \text { i.e., } \omega=3 \omega^{\prime}+\beta, \beta \in \Delta(G), \omega^{\prime} \text { weight, } \\ \underline{\text { type (b) }} & n \equiv m \equiv n-m \equiv 0(\bmod 3) \\ & \text { i.e., } \omega=3 \omega^{\prime} \text { for some weight } \omega^{\prime}, \\ \underline{\text { type (c) }} & \text { Exactly one of } n, m, n-m \text { is } \equiv 0(\bmod 3) .\end{cases}
$$

Here types (a) and (b) give $R_{G} . W=S_{3}$ permutes the $\theta^{\prime}$, and we split $\Omega^{\prime}\left(X^{\prime}\right)$ into $S_{3}$-orbits of pairs $( \pm \omega)$

$$
\Omega^{\prime}\left(X^{\prime}\right)=\sum_{k} d_{k} \cdot \Delta_{k}+\sum_{k} l_{3 k} \cdot \Lambda_{3 k}+\left[\Gamma_{1}+\Gamma_{2}+\cdots\right],
$$

where $\Delta_{k}=\left\{ \pm k\left(\theta_{i}-\theta_{j}\right)\right\}$, e.g., $\Delta_{1}=\Delta(G), \Lambda_{l}=\left\{ \pm l \theta_{i}\right\}, k, l>0$, and each $\Gamma_{i}$ is different from $\Delta_{k}$ or $\Lambda_{3 k}$. Define the associated numbers of (18),

$$
\left\{\begin{array}{l}
\delta=\sum d_{k}, \delta_{1}=\sum d_{\text {odd }}, \delta_{2}=\sum d_{\text {even }}, \delta_{3}=\sum d_{3 k}, \\
\gamma_{\pi}=\#\left\{\Gamma_{i} ; \Gamma_{i} \text { of type }(\pi) \text { in }(18)\right\}, \pi=a, b, \\
s_{a}=\delta-\delta_{3}+2 \gamma_{a}, s_{b}=\delta_{3}+\sum l_{3 k}+2 \gamma_{b} .
\end{array}\right.
$$

PROPOSITION 4.1. Let $X \sim \mathbf{z}_{6}$ pt or $S^{N}$ be an $S U(3)$-manifold with $X^{T} \neq \varnothing$, and let (18) be the collection of nonzero weights lying in the root lattice. Then the effective Weyl group $\bar{W}$, acting on $X^{T}$, is determined by (19) as follows:

(i) $\bar{W}=S_{3} \Leftrightarrow\left[s_{a} \neq 2 s_{b}, \delta_{1}>\delta_{2}\right]$,

(ii) $\bar{W}=S_{3} / \mathbf{Z}_{3} \Leftrightarrow\left[s_{a}=2 s_{b}, \delta_{1}>\delta_{2}\right]$,

(iii) $\bar{W}=1 \Leftrightarrow\left[s_{a}=2 s_{b}, \delta_{1}=\delta_{2}\right]$.

ProOF. Let $\langle C\rangle \simeq \mathbf{Z}_{3}$ in $S U(3)$, where

$$
C=\left(\begin{array}{lll}
0 & 0 & 1 \\
1 & 0 & 0 \\
0 & 1 & 0
\end{array}\right) \sim\left(\begin{array}{ccc}
\xi & 0 & 0 \\
0 & \xi^{-1} & 0 \\
0 & 0 & 1
\end{array}\right)=t_{3} \in T, \quad \xi=e^{2 \pi i / 3}
$$

and $N_{3}=\langle T, C\rangle \subset N(T)$. We shall assume (for simplicity) that $X^{N_{3}} \neq \varnothing$, e.g., $X$ is acyclic. (If $X^{N_{3}}=\varnothing$, then clearly $\bar{W}=S_{3}$.) Let $X_{x}^{H}$ be the space fixed by 
$H \subset N_{3}$ in the local representation at $x \in X^{N_{3}}$, and decompose

$$
\left\{\begin{array}{l}
X_{x}=\left[X_{x}^{N_{3}} \oplus V\right] \oplus \sum \mathbf{R}_{\nu}^{6}=X_{x}^{T} \oplus \sum \mathbf{R}_{\nu}^{6} \\
X_{x}^{\langle C\rangle}=X_{x}^{N_{3}} \oplus \sum_{\nu}\left(\Delta \mathbf{R}^{2}\right)_{\nu}
\end{array}\right.
$$

Here $V$ is a sum of 2-dimensional representations of $\mathbf{Z}_{3}=N_{3} / T, \mathbf{R}_{\nu}^{6}=\mathbf{R}_{ \pm \omega_{i}}^{2} \oplus$ $\mathbf{R}_{ \pm \omega_{j}}^{2} \oplus \mathbf{R}_{ \pm \omega_{k}}^{2}$ is a sum of weight spaces of a $\mathbf{Z}_{3}$-orbit $\nu$ of weight pairs, $\nu=$ $\left\{ \pm \omega_{i}, \pm \omega_{j}, \pm \omega_{k}\right\},\left(\Delta \mathbf{R}^{2}\right)_{\nu} \simeq \mathbf{R}^{2}$ is the "diagonal" in $\mathbf{R}_{\nu}^{6}$ under the cyclic permutation of the three summands, induced by $C$. Since $C \sim t_{3}$, we obtain identities

$$
\begin{aligned}
\operatorname{dim} X^{\langle C\rangle} & =\operatorname{dim} X^{N_{3}}+2 \cdot \#\left\{\mathbf{Z}_{3} \text {-orbits of pairs }( \pm \omega) \in \Omega^{\prime}(X)\right\} \\
& =\operatorname{dim} X^{\left\langle t_{3}\right\rangle}=\operatorname{dim} X^{T}+2 \cdot \#\left\{( \pm \omega) \in \Omega^{\prime}(X) ; \omega\left(t_{3}\right) \in \mathbf{Z}\right\}
\end{aligned}
$$

and hence,

$$
X^{T}=X^{N_{3}} \Leftrightarrow \#\left\{\mathbf{Z}_{3} \text {-orbits in } \Omega^{\prime}(X)\right\}=\#\left\{( \pm \omega) \in \Omega^{\prime}(X) ; \omega\left(t_{3}\right) \in \mathbf{Z}\right\} .
$$

Now, since $\Delta_{k}$ and $\Lambda_{k}$ are both $S_{3}$-orbits and $\mathbf{Z}_{3}$-orbits, whereas each $\Gamma_{i}$ in (18) splits into two $\mathbf{Z}_{3}$-orbits, we find that if we define

$$
s_{\pi}=\#\left\{\mathbf{Z}_{3} \text {-orbits of type }(\pi) \text { in } \Omega^{\prime}(X)\right\}, \quad \pi=a, b, c,
$$

then (24) agrees with (19) for $\pi=a, b$. Let $q$ be the number of pairs $\left( \pm \omega_{i}\right)$ in $\mathbf{Z}_{3}\{ \pm \omega\}$ such that $\omega_{i}\left(t_{3}\right) \in \mathbf{Z}$. Then $q=0,3,1$ for $\omega$ of type (a), (b), (c) respectively. By (22), $s_{a}+s_{b}+s_{c} \geq 3 s_{b}+s_{c}$, and the right side of (23) is equivalent to $s_{a}+s_{b}+s_{c}=3 s_{b}+s_{c}$. Consequently, (23) may be written

$$
\bar{W} \neq S_{3} \Leftrightarrow s_{a}=2 s_{b},
$$

and combining this with the results in $\S 3(\mathrm{C})$ completes the proof.

REMARKS 4.2. (i) The cancelling of $s_{c}$ in the above relations reflects the fact that weights of type $(c)$ in $\Omega(X)$ are irrelevant for $\bar{W}$.

(ii) From the proof it also follows that $s_{a} \geq 2 s_{b}$ (at least if $F\left(\mathbf{Z}_{3}, X^{T}\right) \neq \varnothing$ ). Moreover, $\delta_{1}=\delta_{2} \Rightarrow s_{a}=2 s_{b}$.

(B) $G=\operatorname{Sp}(2)$. Consider $\operatorname{Sp}(2)$ as quaternionic unitary $2 \times 2$-matrices, with $T=\operatorname{diag}\left\langle e^{2 \pi i \theta_{1}}, e^{2 \pi i \theta_{2}}\right\rangle$ and dihedral Weyl group

$$
W=\left\{a^{j} b^{i} ; a^{2}=b^{4}=1, a b a=b^{-1}\right\}=D_{4}
$$

containing two nonconjugate groups $\simeq D_{2}$, namely

$$
D_{2}=W_{\mathrm{Sp}(1) \times \mathrm{Sp}(1)}=\left\{1, b^{2}, a, a b^{2}\right\}, \quad D_{2}^{\prime}=\left\{1, b^{2}, a b, a b^{3}\right\} .
$$

$W$ acts on weights $\omega=\left(n \theta_{1}+m \theta_{2}\right)$ by (i) $b \theta_{1}=-\theta_{2}, b \theta_{2}=\theta_{1}$, (ii) $a \theta_{1}=-\theta_{1}$, $a \theta_{2}=\theta_{2}$. Also recall that $G$ has root system $\Delta(G)=\left\{ \pm\left(\theta_{1} \pm \theta_{2}\right), \pm 2 \theta_{1}, \pm 2 \theta_{2}\right\}$, and hence root lattice

$$
R_{G}: \omega=\left(n \theta_{1}+m \theta_{2} ; \quad n+m \equiv 0 \quad(\bmod 2) .\right.
$$

Now, given $\Omega(X),(16)$, we shall write $\Omega^{\prime}(X) \cap R_{G}$ as

$$
\Omega^{\prime}\left(X^{\prime}\right)=\sum_{k} d_{k}\left\{ \pm k\left(\theta_{1} \pm \theta_{2}\right)\right\}+\sum_{k} e_{k}\left\{ \pm k\left(2 \theta_{i}\right)\right\}+\Gamma
$$


where $\Gamma$ does not contain any root multiple $k \alpha, k \geq 1$. The associated numbers of (26) are defined by counting pairs $( \pm \omega)$, namely

$$
\left\{\begin{array}{l}
\delta_{1}=\sum d_{\mathrm{odd}}, \delta_{2}=\sum d_{\mathrm{even}}, \varepsilon_{1}=\sum e_{\mathrm{odd}}, \varepsilon_{2}=\sum e_{\mathrm{even}} \\
\left.\lambda_{2}=\#\left\{( \pm \omega) \in \Omega^{\prime}\left(X^{\prime}\right)\right\}, \text { i.e., } n+m \equiv 0(\bmod 2)\right\} \\
\lambda_{4}=\#\left\{( \pm \omega) \in \Omega^{\prime}\left(X^{\prime}\right) ; n+m \equiv 0(\bmod 4)\right\} \\
\lambda=\#\left\{( \pm \omega) \in \Omega^{\prime}\left(X^{\prime}\right) ; n+5 m \equiv 0(\bmod 8)\right\}
\end{array}\right.
$$

Proposition 4.3. Let $\operatorname{Sp}(2)$ act on $X \sim \mathbf{z}_{2}$ pt or $S^{N}, X^{T} \neq \varnothing$, and let (26) be those weights in $\Omega^{\prime}(X)$ lying in the root lattice (25). Then the effective Weyl group, $\bar{W}$, is determined by the associated numbers, (27), as follows:

(i) $\bar{W}=D_{4} \Leftrightarrow\left[2 \lambda_{4}<\lambda_{2}>4 \lambda, \delta_{1}>\delta_{2}, \varepsilon_{1}>\varepsilon_{2}\right]$,

(ii) $\bar{W}=D_{4} / \mathbf{Z}_{2} \Leftrightarrow\left[2 \lambda_{4}=\lambda_{2}>4 \lambda, \delta_{1}>\delta_{2}, \varepsilon_{1}>\varepsilon_{2}\right]$,

(iii) $\bar{W}=D_{4} / \mathbf{Z}_{4} \Leftrightarrow\left[2 \lambda_{4}=\lambda_{2}=4 \lambda, \delta_{1}>\delta_{2}, \varepsilon_{1}>\varepsilon_{2}\right]$,

(iv) $\bar{W}=D_{4} / D_{2} \Leftrightarrow\left[2 \lambda_{4}=\lambda_{2}>4 \lambda, \delta_{1}>\delta_{2}, \varepsilon_{1}=\varepsilon_{2}\right]$,

(v) $\bar{W}=D_{4} / D_{2}^{\prime} \Leftrightarrow\left[2 \lambda_{4}=\lambda_{2}>4 \lambda, \delta_{1}=\delta_{2}, \varepsilon_{1}>\varepsilon_{2}\right]$,

(vi) $\bar{W}=1 \Leftrightarrow\left[2 \lambda_{4}=\lambda_{2}=4 \lambda, \delta_{1}=\delta_{2}, \varepsilon_{1}=\varepsilon_{2}\right]$.

The proof is based upon two lemmas. First, similar to (20), choose elements $A, B \in N=N(T)$ representing $a, b \in W$ :

$$
A=\left(\begin{array}{ll}
j & 0 \\
0 & 1
\end{array}\right), \quad B=\left(\begin{array}{ll}
0 & j \\
1 & 0
\end{array}\right) \sim\left(\begin{array}{cc}
\xi & 0 \\
0 & -\xi
\end{array}\right)=t_{8} \in T, \quad \xi=e^{2 \pi i / 8},
$$

and let $N_{a}=\langle T, A\rangle, N_{b}=\langle T, B\rangle$, etc. be subgroups of $N$.

LEMMA 4.4. Let $\psi$ be an irreducible real representation of $N_{c}, c=b, b^{2}$, such that $\psi \mid T$ is nontrivial. Then $\operatorname{dim} \psi=4$ or 2 for $c=b$ or $b^{2}$ respectively, and with $C=B$ or $B^{2}$ :

$$
\operatorname{dim} F(C, V(\psi))= \begin{cases}1, & \text { if } \Omega(\psi \mid T) \subset R_{G} ; \quad c f .(25), \\ 0, & \text { otherwise. }\end{cases}
$$

ProOF OF 4.4. The two cases are similar, so assume $c=b$ and write $\langle b\rangle=\mathbf{Z}_{4} \subset$ $W$. Any $\mathbf{Z}_{4}$-orbit of $T$-weight pairs consists of two pairs; $\Omega(\psi \mid T)=\left\{ \pm\left(n \theta_{1}+m \theta_{2}\right)\right.$, $\left.\pm\left(n \theta_{2}-m \theta_{1}\right)\right\}$ is such an orbit. Let $v_{i}, 1 \leq i \leq 4$, be a basis for the four complex weight lines of $\psi \otimes \mathbf{C}$. $B$ permutes the lines cyclically, say, $B v_{i}=v_{i+1}, i=1,2,3$, and $B v_{4}=B^{4} v_{1}=e^{2 \pi i(n+m) / 2} \cdot v_{1}$ since $B^{4}=\operatorname{diag}\langle-1,-1\rangle \in T,(28)$. From this it is clear that $\operatorname{dim} F(B, V(\psi))=1$ if $n+m \equiv 0(\bmod 2)$, and is zero otherwise.

LEMMA 4.5. Let $c \in\left\{a, a b, b, b^{2}\right\} \subset D_{4}$ and assume $F\left(c, X^{T}\right) \neq \varnothing, \bar{W}=$ $D_{4} / W_{0}$. The following relations hold:

(i) $c=b: b \in W_{0} \Leftrightarrow \lambda_{2}=4 \lambda$,

(ii) $c=b^{2}: b^{2} \in W_{0} \Leftrightarrow \lambda_{2}=2 \lambda_{4}$,

(iii) $c=a: a \in W_{0} \Leftrightarrow D_{2} \subset W_{0} \Leftrightarrow \varepsilon_{1}=\varepsilon_{2}$,

(iv) $c=a b: a b \in W_{0} \Leftrightarrow D_{2}^{\prime} \subset W_{0} \Leftrightarrow \delta_{1}=\delta_{2}$.

ProOF OF 4.5. Since $c \in W_{0} \Leftrightarrow X^{T}=X^{N_{c}}$, and ord $c=2$ or 4 , by Smith theory it suffices to consider the local representation of $N_{c}$ at some fixed point $x \in X^{N_{c}}$, namely

$$
X_{x}=X_{x}^{T} \oplus \sum \mathbf{R}_{ \pm \omega}^{2}=\left[X_{x}^{N_{c}} \oplus V\right] \oplus \sum \mathbf{R}_{ \pm \omega}^{2} .
$$


Here $V \subset X_{x}^{T}$ is the (orthogonal) complement of the space fixed by $c$, hence $V=$ $0 \Leftrightarrow c \in W_{0}$. Now, by Lemma 4.4 , we have on the one hand

$$
\operatorname{dim} X^{\langle C\rangle}=\operatorname{dim} X^{N_{c}}+ \begin{cases}\lambda_{2} / 2, & C=B, \\ \lambda_{2}, & C=B^{2},\end{cases}
$$

but since $B \sim t_{8},(28)$, we have on the other hand

$$
\operatorname{dim} X^{\langle C\rangle}=\operatorname{dim} X^{T}+ \begin{cases}2 \lambda, & C=B, \\ 2 \lambda_{4}, & C=B^{2} .\end{cases}
$$

This proves cases (i) and (ii).

It is clear that $a \in W_{0} \Rightarrow b^{2} \in W_{0}$. Finally, note that $\langle a\rangle=W_{\alpha},\langle a b\rangle=W_{\beta}$, where $\alpha=2 \theta_{1}, \beta=\theta_{1}+\theta_{2}$ in $\Delta(G)$. Therefore (iii), (iv) follow by looking at $S O(3)$-actions $\left(\bar{G}_{\alpha}, X_{\alpha}\right),\left(\bar{G}_{\beta}, X_{\beta}\right)$, cf. $\S 3(C)$.

ProOF OF 4.3. If some $\gamma \in W$ vanishes in $\bar{W}$, then $F\left(\gamma, X^{T}\right)=X^{T} \neq \varnothing$. By Proposition $3.7 \delta_{1} \geq \delta_{2}, \varepsilon_{1} \geq \varepsilon_{2}$, so the implications " $\Rightarrow$ " follow from Lemma 4.5, at least if inequalities " $<$ " among $\lambda$ 's are replaced by " $\neq$ ". However, $\lambda_{2} \geq 2 \lambda_{4}$, $\lambda_{2} \geq 4 \lambda$ clearly hold unless $X \sim S^{N}$ and $F\left(D_{4}, X^{T}\right)=\varnothing$. But $D_{4}$-actions on $Y \sim \mathbf{z}_{2} S^{r}$ are always cohomologically modeled after linear actions (cf. [10, Theorem 4.6], so the appropriate dimensional relations among fixed point sets of subgroups of $D_{4}$ will hold in any case. Finally, the implications can be replaced by equivalences, since the right sides are mutually exclusive.

REMARK 4.6. (i) in 4.3 can be replaced by $\bar{W}=D_{4} \Leftrightarrow \lambda_{2}>2 \lambda_{4}$. More consistency conditions on $\Omega^{\prime}\left(X^{\prime}\right)$ can be found; for example, by Proposition 3.7 the right sides in (iv)-(vi) can be shortened so that only $\delta_{1}, \delta_{2}, \varepsilon_{1}, \varepsilon_{2}$ are involved-the $\lambda$-type relations are therefore implied by the others.

(C) $G=S U(2) \times S U(2)$. Since we work in the root lattice, we may as well assume $\overline{G=S O(3) \times S O(3)}$ acts on $X$,

$$
\Omega^{\prime}(X)=\sum_{k} d_{k}\{ \pm k \alpha\}+\sum_{k} e_{k}\{ \pm k \beta\}+\Gamma
$$

$\Delta(G)=\{ \pm \alpha, \pm \beta\}$, and $\Gamma$ consists of the "mixed" weights $\omega=n \alpha+m \beta$, both $n, m \neq 0$. The splitting of $W=D_{2}=\mathbf{Z}_{2} \times \mathbf{Z}_{2}$ refers to the factors of $G$. The associated numbers of (29) are

$$
\left\{\begin{array}{l}
\delta_{i}, \varepsilon_{i} \text { as in }(27) \\
\mu=\#\left\{( \pm \omega) \in \Omega^{\prime}(X)\right\} \\
\mu_{2}=\#\left\{( \pm \omega) \in \Omega^{\prime}(X) ; \omega=n \alpha+m \beta, n+m \equiv 0(\bmod 2)\right\}
\end{array}\right.
$$

PROPOSITION 4.7. Let $S O(3) \times S O(3)$ act on $X \sim \mathbf{z}_{2}$ pt or $S^{N}$ with $X^{T} \neq \varnothing$ and nonzero weights given by (29). Then $\bar{W}$ is determined from (30), as follows:

(i) $\bar{W}=D_{2} \Leftrightarrow\left[\mu>2 \mu_{2}, \delta_{1}>\delta_{2}, \varepsilon_{1}>\varepsilon_{2}\right]$,

(ii) $\bar{W}=D_{2} / \Delta \mathbf{Z}_{2} \Leftrightarrow\left[\mu=2 \mu_{2}, \delta_{1}>\delta_{2}, \varepsilon_{1}>\varepsilon_{2}\right]$,

(iii) $\bar{W}=D_{2} / \mathbf{Z}_{2} \times 1 \Leftrightarrow\left[\delta_{1}=\delta_{2}, \varepsilon_{1}>\varepsilon_{2}\right]$,

(iv) $\bar{W}=D_{2} / 1 \times \mathbf{Z}_{2} \Leftrightarrow\left[\delta_{1}>\delta_{2}, \varepsilon_{1}=\varepsilon_{2}\right]$,

(v) $\bar{W}=1 \Leftrightarrow\left[\delta_{1}=\delta_{2}, \varepsilon_{1}=\varepsilon_{2}\right] \Rightarrow\left[\mu=2 \mu_{2}\right]$.

(D) $G=G_{2}$. Let $T \subset S U(3) \subset G_{2}$; here $T$ is the maximal torus parametrized as in (A), and hence we write

$$
\Delta\left(G_{2}\right)=\Delta(S U(3))+\Omega^{\prime}\left(\rho_{7}\right)=\left\{ \pm\left(\theta_{i}-\theta_{j}\right)\right\}+\left\{ \pm \theta_{i}\right\}
$$




$$
\Omega^{\prime}(X)=\sum_{k} d_{k}\left\{ \pm k \theta_{i}\right\}+\sum_{k} e_{k}\left\{ \pm k\left(\theta_{i}-\theta_{j}\right)\right\}+\Gamma,
$$

where $\Gamma$ contains no root multiple. Put

$$
\begin{aligned}
& W=D_{6}=\left\{a^{j} b^{i} ; a^{2}=b^{6}=1, a b a=b^{-1}\right\}, \\
& W_{S U(3)}=D_{3}=\left\{1, b^{2}, b^{4}, a, a b^{2}, a b^{4}\right\} \sim D_{3}^{\prime}, \\
& W_{S O(4)}=D_{2}=\left\{1, b^{3}, a, b^{3}\right\},
\end{aligned}
$$

$$
\mathcal{R}_{S O(4)}: \omega=n \theta_{1}+m \theta_{2}, n+m \equiv 0 \quad(\bmod 2) \quad\left(\text { note! } \theta_{3}=-\theta_{1}-\theta_{2}\right),
$$

$$
\left\{\begin{array}{l}
\delta_{1}, \delta_{2}, \varepsilon_{1}, \varepsilon_{2} \text { as in }(27) \\
\mu=\#\left\{( \pm \omega) \in \Omega^{\prime}(X) \cap R_{S O(4)}\right\} \\
\mu_{2}=\#\left\{( \pm \omega) \in \Omega^{\prime}(X) \cap R_{S O(4)} ; n \equiv m \equiv 0(\bmod 2), \text { cf. }(32)\right\} \\
s_{a}=\sum_{k \neq 0(\bmod 3)} e_{k}+2 \cdot \#\left\{\text { orbits } D_{3}( \pm \omega) \text { in } \Gamma ; \omega \text { of type }(a) \text { in }(17)\right\} \\
s_{b}=\sum_{k}\left(d_{3 k}+e_{3 k}\right)+2 \cdot \#\left\{\text { orbits } D_{3}( \pm \omega) \text { in } \Gamma ; \omega \text { of type }(b) \text { in }(17)\right\}
\end{array}\right.
$$

Note that $b^{2} \in D_{3}$ survives in $\bar{W}$ iff it does so in $\bar{D}_{3}$ (for $X \mid S U(3)$ ); and $b^{3}$ survives iff it survives in $\bar{D}_{2}$ (for $X \mid S O(4)$ ). So by suitably combining results from (A), (C), the next proposition (as well as more consistency conditions, which we omit), can be established.

Proposition 4.8. Let $X \sim \mathbf{z}_{6}$ pt or $S^{N}$ be a $G_{2}$-manifold, $X^{T} \neq \varnothing$ and nonzero weights given by (31). Then $\bar{W}$ can be calculated from (33), as follows:

(i) $\bar{W}=D_{6} \Leftrightarrow\left[s_{a} \neq 2 s_{b}, \mu>2 \mu_{2}, \delta_{1}>\delta_{2}, \varepsilon_{1}>\varepsilon_{2}\right]$,

(ii) $\bar{W}=D_{6} / \mathbf{Z}_{2} \Leftrightarrow\left[s_{a} \neq 2 s_{b}, \mu=2 \mu_{2}, \delta_{1}>\delta_{2}, \varepsilon_{1}>\varepsilon_{2}\right]$,

(iii) $\bar{W}=D_{6} / \mathbf{Z}_{3} \Leftrightarrow\left[s_{a}=2 s_{b}, \mu>2 \mu_{2}, \delta_{1}>\delta_{2}, \varepsilon_{1}>\varepsilon_{2}\right]$,

(iv) $\bar{W}=D_{6} / \mathbf{Z}_{6} \Leftrightarrow\left[s_{a}=2 s_{b}, \mu=2 \mu_{2}, \delta_{1}>\delta_{2}, \varepsilon_{1}>\varepsilon_{2}\right]$,

(v) $\bar{W}=D_{6} / D_{3} \Leftrightarrow\left[s_{a}=2 s_{b}, \mu>2 \mu_{2}, \delta_{1}>\delta_{2}, \varepsilon_{1}=\varepsilon_{2}\right]$,

(vi) $\bar{W}=D_{6} / D_{3}^{\prime} \Leftrightarrow\left[s_{a}=2 s_{b}, \mu>2 \mu_{2}, \delta_{1}=\delta_{2}, \varepsilon_{1}>\varepsilon_{2}\right]$,

(vii) $\bar{W}=1 \Leftrightarrow\left[s_{a}=2 s_{b}, \mu=2 \mu_{2}, \delta_{1}=\delta_{2}, \varepsilon_{1}=\varepsilon_{2}\right]$.

(E) On the estimation of $\delta(G)$. In [7] the following numerical invariant of compact Lie groups $G$ is studied:

$$
\tilde{\delta}(G)=\min \{\operatorname{dim} X / G ; X: \mathbf{Z} \text {-acyclic effective } G \text {-manifold without fixed point }\}
$$

In this subsection we shall apply the results in (A), (B) to improve the estimates of $\hat{\delta}(G)$ in $[7]$ for $G=S O(5)$ and $S U(3)$.

First assume $G=S U(3) / \mathbf{Z}_{3}$, acting with no fixed point on $X \sim \mathbf{z}_{3}$ pt, and with $F(T) \mathrm{Z}$-acyclic. By $[\mathbf{7}, \S 3] \delta \geq 2$ in (19), and $\Omega^{\prime}(X)$ must contain weights of type (b) in (17). Moreover, the simplest possibility of weight pattern is $\Omega^{\prime}(X)=$ $2 \Delta_{1}+\left\{ \pm 3 \theta_{i}\right\}$. However, this weight pattern gives $\bar{W}=\mathbf{Z}_{2}$ by Proposition 4.1, hence $F(G)=F\left(W, X^{T}\right) \neq \varnothing$, cf. Proposition 3.7(iii). Similarly, other cases with $\delta=2$ can be ruled out. The simplest $G$-weight pattern, possibly realizable with $F(G)=\varnothing$, is now

$$
\Omega=2 \Delta_{1}+\Delta_{2}+\left\{ \pm 3 \theta_{i}\right\}+m_{0}\{0\}, \quad m_{0} \geq 2 .
$$

Consequently, $\tilde{\delta}\left(S U(3) / Z_{3}\right) \geq 18, \tilde{\delta}(S U(3)) \geq 24$, cf. [7, 3.4]. 
Next, let $G=S O(5)$. Consider the weight pattern $\Omega^{\prime}=2\left\{ \pm \alpha_{i}\right\}+\left\{ \pm 2 \alpha_{i}\right\}+$ $\left\{ \pm \beta_{i}\right\}+\left\{ \pm 2 \beta_{i}\right\}, \alpha_{i}=$ short root, $\beta_{i}=$ long root. This gives $\lambda_{2}=10, \lambda_{4}=6$ in (27), namely $\lambda_{2}<2 \lambda_{4}$, so $\Omega^{\prime}$ cannot be realizable, by Proposition 4.3. Hence, following $[\mathbf{7}, 3.4]$

$$
\Omega=\Omega^{\prime}\left(2 S^{2} \rho_{5}\right)+m_{0}\{0\}, \quad 2 \leq m_{0} \leq 5,
$$

is, indeed, a "minimal model" $G$-weight pattern for acyclic $G$-manifolds $X$ with no fixed point. Consequently, $16 \leq \tilde{\delta}(S O(5)) \leq 19$.

(F) Regularity as a consequence of the $G$-weight pattern $d \cdot \Delta(G)$. In this subsection we shall prove two theorems about $G$-manifolds with orbit structure closely related to that of $d \cdot \operatorname{Ad}_{G}$. We assume $X$ is acyclic or spherical mod $\mathbf{Z}$, although technically we need only coefficients $\mathbf{Z}_{q}, q=$ product of the prime factors of $|W|$.

THEOREM 4.9. Let $G$ be a semisimple group of rank 2 acting on $X \sim \mathrm{pt}$ or $S^{N}$, and assume the nonzero geometric weight system satisfies

$$
\Omega(X)=\Omega\left(X^{\prime}\right)+\Gamma, \quad \Omega^{\prime}\left(X^{\prime}\right) \subset d \cdot \Delta(G),
$$

where $\Gamma$ consists of those weights outside the root lattice. Then

(i) for each connected $H, T \subset H \subset G$,

$$
F(H)=F\left(W_{H}, X^{T}\right)=F\left(c_{H}, X^{T}\right),
$$

where $c_{H} \in W_{H}$ is a Coxeter element;

(ii) $W$ acts effectively on $X^{T}$, i.e., $\bar{W} \simeq W$, if and only if $\Delta(G) \subset \Omega^{\prime}(X)$.

PROOF. (ii) follows from Propositions 4.1, 4.3, 4.7 and 4.8, and the first equality in (35) is clear, cf. Proposition 3.7(iii).

Assume rk $W_{H}=2, H \subset G$, and note that $X \mid H$ satisfies the $H$-version of (34) since each $\alpha \in \Delta(G)-\Delta(H)$ is outside the lattice $R_{H}$. Let $C_{H} \in H$ represent $c_{H} \in$ $W_{H}$ and let $t_{H} \in T$ be a conjugate image; for $H \neq G_{2}$ explicit expressions for $t_{H}$ are easy to find (e.g. $t_{3}, t_{8}$ in (20), (28)). Since $H$ acts on $F(Z(H), X)$ with weight system $\Omega^{\prime}\left((X \mid H)^{\prime}\right) \subset k \Delta(H)$, comparison of dimensions gives $F\left(t_{H}\right)=F(T)$, that is, $F\left(C_{H}\right)=F\left(T^{\prime}\right)$ for some $T^{\prime} \ni C_{H}$. But no proper $W_{K} \subset W_{H}$ contains $c_{H}$, for $T \subset K \subset H$ connected, so $\left\langle T, T^{\prime}\right\rangle=H$,

$$
F\left(c_{H}, F(T)\right)=F\left(C_{H}\right) \cap F(T)=F\left(T^{\prime}\right) \cap F(T)=F(H) .
$$

Finally, let $H=G_{2}$. One can choose $C_{H}$ such that $C_{H}^{2}=C \in S U(3)$ in (20), and moreover, $C_{H} \sim t_{H} \in T, t_{H}^{2}=t_{3}$. Since $\Gamma=\varnothing$, we can find some circle group $S, t_{H} \in S \subset T$, so that $F\left(t_{H}\right)=F(S)$. Now the above argument giving (36) can be used again.

An interesting special case of Theorem 4.9 is $\Omega^{\prime}(X)=d \cdot \Delta(G)$. In the linear case, $d \cdot \operatorname{Ad}_{G}, W$ acts diagonally on $F(T)=\tau \oplus \tau \oplus \cdots(d$ copies $)$, and for any subgroup $A \subset W$ with parabolic closure $\tilde{A}$,

$$
F(A, F(T))=F(\tilde{A}, F(T)),
$$

because $O\left(d \cdot \overline{\operatorname{Ad}}_{G}\right)=\mathcal{O}\left(\overline{\operatorname{Ad}}_{G}\right)=\left\{\right.$ parabolic $\left.W^{\prime} \subset W\right\}$, cf. (7). (37) will be the clue to the proof of the regularity Theorem 4.11 below. 
LEMMA 4.10. Assume $\Omega^{\prime}\left(X^{\prime}\right)=d \cdot \Delta(G)$. Then the equality (37) holds for the action of $W$ on $F(T, X)$. [This also holds when $\operatorname{rk} G>2$.]

ProOF OF 4.10. We prove the case $\operatorname{rk} G=2$, i.e., $W$ is dihedral, and may assume $\tilde{A}=W$. Note first that $A$ contains some power $c^{l} \neq 1$ of the Coxeter element $c \in W$. (37) will follow if we can show $F\left(c^{l}, X^{T}\right)=F\left(W, X^{T}\right)$. By Theorem 4.9 the remaining cases are (i) $W=D_{4}, l=2$; (ii) $W=D_{6}, l=2$, (iii) $W=D_{6}, l=3$.

In case (iii) let $H=S O(4) \subset G_{2}$, and note that $c_{H}=c^{3}$ is a common Coxeter element for three groups $D_{2} \sim D_{2}^{\prime} \sim D_{2}^{\prime \prime}$. By $(35), F\left(D_{6}, X^{T}\right)=F\left(D_{2}, X^{T}\right)=$ $F\left(c^{3}, X^{T}\right)$. In cases (i), (ii) we have at least $F\left(c^{2}\right)=F\left(D_{2}\right), F\left(D_{3}\right)$ respectively. We shall complete (i) and (ii), assuming $F(W)=\varnothing$, as we may by local linearity. So, assume $X^{T} \sim S^{r}, F\left(c^{2}\right) \sim S^{x}$, and observe that $F\left(\mathbf{Z}_{2}\right) \sim S^{r-d}$ for each $\mathbf{Z}_{2} \not \subset\langle c\rangle$ (cf. Theorem 3.6). It remains to show $x=-1$.

Case (i). Let $D_{2}^{\prime} \neq D_{2}$ in $D_{4}$. Since $F\left(D_{2}\right)=F\left(c^{2}\right) \supset F\left(D_{2}^{\prime}\right), F\left(D_{2}^{\prime}\right)=$ $F\left(D_{4}\right)=\varnothing$. Now Borel's formula (cf. [1, Chapter XIII]) applied to the action of the $\mathrm{Z}_{2}$-tori $D_{2}, D_{2}^{\prime}$ on $X^{T}$ gives two equations in $x, r$ with solution $r=2 d-1$, $x=-1$.

Case (ii). $D_{6}$ acts on $X^{T}$ with $F\left(\mathbf{Z}_{6}\right)=\varnothing$. Let $\mathbf{Z}_{2}, \mathbf{Z}_{2}^{\prime} \not \subset \mathbf{Z}_{6}$ generate $D_{6}$. By a suitable combination of Smith theory and an idea due to Milnor, one can show that $F\left(\mathbf{Z}_{2}\right)$ and $F\left(\mathbf{Z}_{2}^{\prime}\right)$ are "linking" in $X^{T}$, i.e., $F\left(\mathbf{Z}_{2}\right) \stackrel{i}{\rightarrow} X^{T}-F\left(\mathbf{Z}_{2}^{\prime}\right)$ induces an isomorphism in cohomology $\left(\bmod \mathbf{Z}_{2}\right)$. This gives $\operatorname{dim} F\left(\mathbf{Z}_{2}\right)=(r-1) / 2$, and again $r=2 d-1$. Now, $F\left(\mathbf{Z}_{3}\right)=F\left(D_{3}\right) \sim S^{x}$, and if we had $x \geq 0$, then by the representation theory of $D_{3}, r-\operatorname{dim} F\left(\mathbf{Z}_{3}\right)=2\left[\operatorname{dim} F\left(\mathbf{Z}_{2}\right)-\operatorname{dim} F\left(D_{3}\right)\right]$. But this equation implies $x=-1$, a contradiction.

THEOREM 4.11. Let $G, X$ be as in Theorem 4.9, and assume the G-action $\varphi$ on $X$ has weight system

$$
\Omega(X)=d \cdot \Delta(G)+\Gamma+d_{0}\{0\}
$$

where $\Gamma$ has no weight in the root lattice. Then $d_{0}=2 d+l, l \geq 0$, and $X$ is modeled after $V(\Phi), \Phi=\operatorname{Ad}_{G}+l \cdot 1$, in the following sense:

(i) The Cartan-Weyl actions $\bar{\varphi}, \bar{\Phi}$ are cohomologically similar, namely, they have the same isotropy types, $\mathcal{O}(\bar{\varphi})=\mathcal{O}(\bar{\Phi})$, and corresponding fixed point sets $F\left(W_{x}, F(T)\right)$ agree up to $\mathbf{Z}_{p}$-cohomology $(p=2$ or 3 ).

(ii) $\varphi$ and $\Phi$ have the same maximal rank isotropy groups, and

$$
F\left(G_{x}\right)=F\left(W_{x}, F(T)\right), \quad \text { for } x \in F(T) .
$$

(iii) Assume $\Gamma=\varnothing$. Then $\varphi$ is orthogonally modeled after $\Phi$, hence $O(X)=$ $\mathcal{O}(V(\Phi))$ (cf. also 4.12(ii)).

ProOF. By Remark 3.8, Lemma 4.10 (and its proof), only the case (iii), $d \geq 2$, needs to be checked. We must show that $\varphi$ and $\Phi$ also have the same isotropy groups of rank $<2$. We now claim that $\varphi$ and $\Phi$ have identical $\mathbf{Z}_{p}$-weight systems for each $p$ (cf. $\S 3(\mathrm{~A})$ ). This will follow from the known facts that $\Omega(\varphi)=\Omega(\Phi)$ and moreover, their maximal rank isotropy types are the same. The nontrivial cases occur when $F(G)=\varnothing$ (i.e., $l=0$ and $X \sim S^{N}$ ), $G=S O(5), G_{2}, S O(3)^{2}$ and $p=2$. The reason is that $T$ does not contain a maximal $\mathbf{Z}_{2}$-torus. However, assuming $G=S O(5)$, for example, by restricting to the isotropy group $S O(3) \times S O(2)$ and 
using $W_{2}$-invariance of $\Omega_{2}^{\prime}(\varphi)$, we find $\Omega_{2}^{\prime}(\varphi)=\Omega_{2}^{\prime}(\Phi)$. (See $\S 5(D)$ for similar calculations.)

Now, with all weight systems "correct" one can show that all isotropy types are also "correct". As an illustration, let $G=S U(3)$. A rank one isotropy group $K$ has maximal torus $\sim\left(\theta_{1}-\theta_{2}\right)^{\perp}=S_{\alpha}$, by 3.5, and using representation theory we find easily $K^{0}=S_{\alpha}$, consequently $S_{\alpha} \subset K \subset N\left(S_{\alpha}\right)=Z_{G}\left(S_{\alpha}\right)=S[U(2) \times U(1)]$. If $K / K^{0}$ has $p$-torsion, then $K$ contains a $\mathbf{Z}_{p}$-torus of rank 2, say $T_{p} \subset T$. However, $F\left(T_{p}\right)=F(T)$, so this is impossible, and hence $K=S_{\alpha}$.

Next, a maximal $\mathbf{Z}_{p}$-torus of any isotropy group in $S U(3)$ must be "correct", i.e., conjugate to $T_{p}$ or $T_{p} \cap S_{\alpha}$, e.g., by the $\mathbf{Z}_{p}$-version of Lemma 3.5. But $F\left(T_{p} \cap S_{\alpha}\right)=$ $F\left(S_{\alpha}\right)$ (resp. $\left.X\right)$ for $p \neq 3$ (resp. $p=3$ ). Hence, $K=Z(S U(3))=\operatorname{ker} \varphi \simeq \mathbf{Z}_{3}$ is the only finite isotropy group.

REMARKS 4.12. (i) As observed in the proof of 4.11(iii), $O(X)$ is independent of $d$ when $d>1$. This is due to a peculiar property of $\operatorname{Ad}_{G}(G$ any compact connected Lie group), namely $O\left(d \cdot \operatorname{Ad}_{G}\right)=O\left(2 \mathrm{Ad}_{G}\right), \forall d \geq 2$. More generally, if $\Psi$ is a $G$-representation, then $O\left(d \cdot \operatorname{Ad}_{G}+e \cdot \Psi\right)=O\left(2 \operatorname{Ad}_{G}+\Psi\right), \forall d \geq 2, e \geq 1$. This fact is an interesting consequence of inductive slice arguments.

(ii) Let $\psi_{n}$ be the standard representation of $G(n)=S U(3), S O(5), \operatorname{Sp}(2)$, or $G=G_{2}$ and $\psi_{n}=\rho_{7}$. Then Theorem 4.11 can be extended as follows: Put $\Phi=d \cdot \operatorname{Ad}_{G}+k \psi_{n}$ and assume $\Omega^{\prime}(X)=\Omega^{\prime}(\Phi)$. Then $X$ is orthogonally modeled after $\Phi+l \cdot 1$, for some $l \geq 0$. See also $\S 5(\mathrm{~F})$, (iv).

5. 2-Parametric actions of classical groups. Let $G=G(n)$ be a classical group, and let $\left(\theta_{1}, \theta_{2}, \ldots\right)$ be standard coordinates of the maximal torus $T$. A $G$ weight pattern $\Omega$ is said to be of (at most) l-parametric type if each weight $\omega \in \Omega$ can be expressed by $l$ (or less) parameters $\theta_{i}$, namely $\omega=\sum_{i=1}^{l} k_{i} \theta_{i}$. Similarly, an action $\varphi$ is $l$-parametric if $\Omega(\varphi)$ is so. The purpose of this section is to provide proofs of Theorems A, B, C in $\S 1$.

In (A) we establish Table I, namely, a description of those linear models satisfying the basic weight pattern condition in Theorem A. The ideas of the proofs will be sketched in (B), and more details are given in (C)--(E).

(A) Description of the linear models. Let $\psi_{n}=\rho_{n}, \mu_{n}$ or $\nu_{n}$ be the standard representations of $S O(n), S U(n)$ or $\mathrm{Sp}(n)$ on $\mathbf{R}^{n}, \mathbf{R}^{2 n}$ or $\mathbf{R}^{4 n}$ respectively. Besides these the only irreducible real nontrivial 2-parametric representations of $G(n)$, $\operatorname{rk} G(n)>2$, are $\Phi_{n}$ (cf. Table I) given by

$$
\operatorname{Ad}_{G}, \quad S^{2} \rho_{n}-1, \quad\left[\Lambda^{2} \mu_{n}\right]_{\mathbf{R}}, \quad\left[S^{2} \mu_{n}\right]_{\mathbf{R}}, \quad \Lambda^{2} \nu_{n}-1 .
$$

Moreover, by taking (two factor) products of the above groups and tensor products of their standard representations, the resulting weight patterns may also be regarded as 2-parametric. However, for simplicity we shall only consider product groups like $G(m) \times G(n)$. Since $k \psi_{n}, k \geq 1$, are the only 1-parametric representations, we obtain all 2-parametric ones, with only one 2-parametric $W$-orbit of weights, by adding $k \psi_{n}$ to $\Phi_{n}$.

All results for $S O(n)$ or $S U(n)$ extend naturally to the case of $O(n)$ or $U(n)$, so normally we shall use freely the notation $G(n)$ in either case. In Table I, which contains ten linear models $\Phi=\Phi_{n}+k \psi_{n}$ as described above, we have also listed the isotropy types of the 2-parametric component $\Phi_{n}$. 
REMARKS 5.1. (i) In Table I $\Delta^{2}\left[G\left(l_{1}\right) \times \cdots \times G\left(l_{q}\right)\right]$ denotes the "(conjugate) diagonal" imbedding of $G\left(l_{1}\right) \times \cdots \times G\left(l_{q}\right)$ into

$$
G(l) \times G(l) \subset[G(l) \times G(m-l)] \times[G(l) \times G(n-l)], \quad \sum l_{i}=l .
$$

(ii) In the case $G=S O(n), n=2 m, m$ even, $O\left(\operatorname{Ad}_{G}\right)$ contains some additional types besides $U\left(l_{1}\right) \times \cdots \times U\left(l_{q}\right) \times S O(n-2 l)\left(0 \leq \sum l_{i}=l \leq m\right)$, namely $U\left(l_{1}\right) \times \cdots \times U\left(l_{q-1}\right) \times U\left(l_{q}\right)^{\prime}\left(l_{i}>1\right.$, all $l_{i}$ even, $\left.\sum l_{i}=m\right)$. The latter is conjugate to $U\left(l_{1}\right) \times \cdots \times U\left(l_{q}\right)$ inside $O(2 m)$, i.e., via the outer automorphism of $S O(2 m)$.

LEMMA 5.2. Let $G(n)=O(n), U(n)$ or $\operatorname{Sp}(n), \psi_{n}$ be the standard representation and $\Phi_{n}=\operatorname{Ad}_{G(n)}, \Lambda^{2} \psi_{n}$ or $S^{2} \psi_{n}(\bmod$ trivial $)$. Then

(i) $O\left(\Phi_{n}+k \psi_{n}\right), k>0$, is given by all groups of type $H \times 1$, where $H \times 1 \subset$ $G(n-d) \times G(d), 0 \leq d \leq n, H \in \mathcal{O}\left(\Phi_{n-d}\right)$.

(ii) Let $G=G(m) \times G(n), m \leq n, k+k^{\prime}>0$. Then $O\left(\psi_{m} \otimes \psi_{n}+k \psi_{m}+k^{\prime} \psi_{n}\right)$ is given by all groups of type

$$
\begin{aligned}
& \Delta^{2}\left[G\left(l_{1}\right) \times G\left(l_{2}\right) \times \cdots \times G\left(l_{q}\right)\right] \cdot[G(r) \times G(s)], \quad q \geq 0, \\
& 0 \leq \sum l_{i} \leq l \leq m ; m-l-k \leq r \leq m-l, n-l-k^{\prime} \leq s \leq n-l,
\end{aligned}
$$

where $\Delta^{2}[\cdots] \times[G(r) \times G(s)] \subset[G(l) \times G(l)] \cdot[G(m-l) \times G(n-l)]=[G(l) \times$ $G(m-l)] \times[G(l) \times G(n-l)]$, and $G(p)=1$ for $p \leq 0$.

PROOF. (i) Let $\Phi=\Phi_{n}+k \psi_{n}$ and observe that $\Phi \mid G(n-1)$ and the slice representation of $G(n-1) \in \mathcal{O}\left(\psi_{n}\right)$ is of the same "type" as $\Phi$. Hence, we first verify the lemma for $n \leq 2$ and then use induction on $n$. Alternatively, one calculates directly $O(\Phi)=O\left(\Phi_{n}\right) \cap O\left(k \psi_{n}\right)$ (intersection in any position) by observing that $H \cap G(q)$ are the isotropy groups of the natural $H$-action on the space $G(n) / G(q)$ of orthonormal (or unitary) $(n-q)$-frames. The proof of (ii) is similar.

(B) An outline of the proofs of Theorems A, B and C. Here we sketch the essential ideas, leaving more details to the later subsections. The representations $\tilde{\Phi}=\Phi+l \cdot 1$ on $V(\tilde{\Phi})=\mathbf{R}^{N+1}$ or $S^{N}$, $\Phi$ listed in Table I, will be regarded as our basic modelseach of the $G$-manifolds $X \sim \mathbf{z} \mathbf{R}^{N+1}$ or $S^{N}$ which can occur in the theorems can be associated to a unique $\tilde{\Phi}$.

A major part of the work is to establish the complete weight pattern of $X$

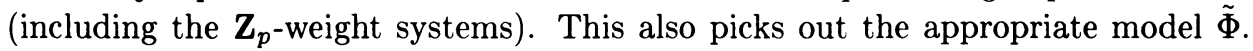
Having all the weight systems, all maximal tori (as well as $\mathbf{Z}_{p}$-tori) of isotropy groups will be "correct" (see e.g. 3.5). From the group structure of classical groups (cf. Theorem 2.2) one can now prove that all connected isotropy groups $G_{x}^{0}$ are "correct", and then the question of $p$-torsion in $G_{x} / G_{x}^{0}$ can be settled-here the

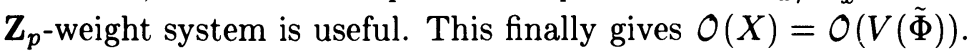

One can also use another approach to establish $O(X)=O(V(\tilde{\Phi}))$, using inductive arguments. Indeed, by restriction to a standard subgroup, say $G(n-1) \subset G(n)$, $\Omega(X \mid G(n-1))$ is precisely of the same "type" as $\Omega(X)$. We also remark that the special case with $k=0$ (in Table I, \#\#1-9) is essentially known (since the principal isotropy type will be nontrivial). However, the value of $k \geq 0$ makes little difference in our proof. In fact, by Lemma 5.2 the orbit types will be the same for all $k \geq 1$. 
Let $H \in O(X)$. It follows from our analysis that, as in the linear model, in most cases the fixed point set $F(H)$ coincides with $F(K)$ for a suitable group $K \subset H$ acting on a suitable subspace $Y \subset X$, such that fixed point theorems of P. A. Smith type hold for $(K, Y)$. Typically, $K$ is a torus, a $p$-group, a group generated by reflections, or an "extension combination" of such groups.

Finally, we remark that the case $F(G) \neq \varnothing$ is somewhat simpler than the case $F(G)=\varnothing$. Therefore, we shall henceforth assume $X \sim S^{N}, F(G)=\varnothing$. Consequently, $l=0$ in the linear models $\tilde{\Phi}=\Phi+l \cdot 1$, and therefore the models encountered in the sequel will be precisely those $\Phi$ in Table I.

(C) Determination of $\Omega(X)$.

As in Theorem $\mathrm{A}$, the basic assumption is on the rational weight system, $\Omega_{0}^{\prime}(X)$, which is defined also when $F(T)=\varnothing$. Assume $\Omega_{0}^{\prime}(X)$ contains no $l$-parametric orbit $W\{ \pm \omega\}$ for $l \geq 3$, and moreover, contains only one 2-parametric orbit. [The case with only 1-parametric orbits is well known, since this will lead to $\Omega(X)=\Omega\left(k \psi_{n}\right)$, cf. [3, I] for manifolds modeled on $k \psi_{n}$.] We claim that the 2-parametric orbit $W\{ \pm \bar{\omega}\}, \bar{\omega}=\left(a \theta_{1}+b \theta_{2}\right)$, must have $|a|=|b|$, namely $\bar{\omega}=\theta_{1} \pm \theta_{2}$. Suppose this is not the case; then $\bar{\omega}$ is not a root, and $S=\bar{\omega}^{\perp}$ is a maximal torus of some isotropy group $G_{x}$ (cf. [5, or 8]). Using (3.1), $G_{x}$ contains the group $H$ generated by all $\bar{G}_{\alpha_{i}}$, $\alpha_{i}=\left(\theta_{r} \pm \theta_{s}\right), r, s>2$. Assume (for simplicity) rk $H \geq 3$ (or $\mathrm{rk} G \geq 5$ ). From the local representation of $H$ it follows that $\bar{\omega} \neq\left(\theta_{1} \pm \theta_{2}\right)$ is impossible, consequently $\Omega_{0}^{\prime}(X)=\Omega_{0}^{\prime}(\Phi)$ for some of our models $\Phi$.

In order to show $\Omega(X)=\Omega(\Phi)$ we shall divide into two main cases.

(i) $\Omega_{0}^{\prime}(X) \cap \Delta(G)=\varnothing$ : This gives $F(T)=F(G)=\varnothing$ (e.g., by (3.1)), and $\Phi$ is \# 2, 3 or 10 in Table I, and we may assume $k>0$. From the local representation of a suitably large isotropy group $\left(G_{x}^{0} \sim S U(n-1), G(m-1) \times G(n)\right.$ or $S O(m-2) \times S O(n))$ we can show that $\Omega(X)$ is well defined, namely $\Omega(X)=\Omega(\Phi)$.

(ii) $\Omega_{0}^{\prime}(X) \cap \Delta(G) \neq \varnothing: \Omega_{0}^{\prime}(X)$ contains precisely one copy of a 2-parametric (rational) root $\bar{\alpha}$, so by considering the $S O(3)$-action $\left(\bar{G}_{\alpha}, X_{\alpha}\right)$, cf. $\S 3$, it follows that $F(T) \neq \varnothing$ and $\alpha \in \Omega^{\prime}(X)$. We may write $\Omega^{\prime}(X)=\Omega^{\prime}(X) \cap \Delta(G)+\Gamma$, where $\Gamma$ is 1-parametric. What can $\Gamma$ be?

Firstly, if $\Delta(G) \subset \Omega^{\prime}(X), G=G(n)$, and $\Gamma$ contains no root multiple, it follows from 3.9 that $W$ acts faithfully on $F(T)$ as a reflection group, and moreover, $F(H)=$ $F\left(W_{H}, F(T)\right), \forall H=H^{0} \supset T$. Since $F(W, F(T))=F(G)=\varnothing, F(T) \sim S^{\text {rk } G-1}$ and $F(H) \neq \varnothing$ for $H=G(n-1)$ or $S O(n-2)$. Again, from the local representation of $H$ it follows that $\Gamma$ must be correct, i.e., $\Gamma=k\left\{ \pm \theta_{i}\right\}, 2 k\left\{ \pm \theta_{i}\right\}, k\left\{ \pm \theta_{i}\right\}$ or $\left\{ \pm 2 \theta_{i}\right\}+k\left\{ \pm \theta_{i}\right\}$, for $G=S U(n), \operatorname{Sp}(n), S O(2 m)$ respectively.

Secondly, if $G=S O(2 m+1)$, then in any case $\Omega^{\prime}(X \mid S O(2 m))=\Omega^{\prime}(X)$, and $X \mid S O(2 m)$ is already known to have weight system of type \#6 or 7 in Table I, hence also of type \#4 or 5 (as $G$-manifold).

Thirdly, let $G=\operatorname{Sp}(n)$ and assume $\Gamma$ contains some $\left\{ \pm 2 l \theta_{i}\right\} . S_{n} \subset W$ maps to a reflection group $\bar{S}_{n} \subset \bar{W}$; the cases $\bar{S}_{n} \simeq S_{2}$ or $\bar{S}_{4}=S_{3}$ can be ruled out, so we assume $\bar{S}_{n} \simeq S_{n}$. Then $F(U(n-1) \times U(1))=F\left(S_{n-1}, F(T)\right) \neq \varnothing$. Similarly, $\bar{W} \simeq S_{n}$ and $F(\operatorname{Sp}(n-1) \times \operatorname{Sp}(1))=F\left(S_{n-1}, F(T)\right) \neq \varnothing$ if $\Gamma$ contains no $\left\{ \pm 2 l \theta_{i}\right\}$. By representation theory, the first case of $\Gamma$ is impossible, and in the last case $\Gamma=2 k\left\{ \pm \theta_{i}\right\}$.

Finally, the case $G=G(m) \times G(n)$ is similar to the above ones. In summary, we now have $\Omega(X)=\Omega(\Phi)$, or at least $\Omega^{\prime}(X)=\Omega^{\prime}(\Phi)$ if $G=S O(2 m+1)$ or 
$S O(m) \times S O(n), m$ or $n$ odd. In these two cases the calculation of zero weight multiplicity, namely $\operatorname{dim} F(T)$, will be done by means of the $\mathbf{Z}_{2}$-weight pattern, see (D) below.

(D) Calculation of $\Omega_{p}(X)$. For $p \neq 2$ the maximal $\mathbf{Z}_{p}$-torus $T_{p}$ in $T$ is also maximal in $G$. Since no $\omega \in \Omega(\Phi)$ is $p$-divisible, $\Omega_{p}(X)=\Omega_{p}(\Phi) \mid T_{p}=\Omega_{p}(\Phi)$ follows from $\Omega(X)=\Omega(\Phi)$. Henceforth, assume $p=2$. (i) Let $G=S O(n), n=2 m$, $\Phi=\operatorname{Ad}_{G}+k \rho_{n}$. By Proposition 3.9 the maximal rank isotropy types in $\mathcal{O}(X)$ are identical to $O\left(\operatorname{Ad}_{G}\right)-\{G\}$. The local representation of $G_{x}=S O(n-2) \times S O(2)$ is $\left(\operatorname{Ad}_{S O(n-2)}+\rho_{n-2} \otimes \rho_{2}+1\right)$. Let

$$
T_{2}=\left\{\operatorname{diag}\left\langle\varepsilon_{1}, \varepsilon_{2}, \ldots, \varepsilon_{n}\right\rangle ; \varepsilon_{i}= \pm 1, \prod \varepsilon_{i}=1\right\}, \quad T_{2}^{\prime}=T_{2} \cap G_{x},
$$

be a maximal $\mathbf{Z}_{2}$-torus in $S O(n)$ (resp. $G_{x}$ ). It follows that

$$
\begin{aligned}
\Omega_{2}^{\prime}(X) \mid T_{2}^{\prime}= & \left\{\left(\varepsilon_{i} \varepsilon_{j}\right) ; i<j \leq n-2\right\} \\
& +\left\{\left(\varepsilon_{i} \varepsilon_{n-1}\right),\left(\varepsilon_{i} \varepsilon_{n}\right) ; i \leq n-2\right\}+k\left\{\varepsilon_{i} ; i \leq n\right\} \\
= & \Omega_{2}^{\prime}\left(\operatorname{Ad}_{G}+k \rho_{n}\right) \mid T_{2}^{\prime} .
\end{aligned}
$$

Since $\Omega_{2}^{\prime}(X)$ is invariant under $W_{2}=N\left(T_{2}\right) / T_{2} \simeq S_{n}$, it follows that $\Omega_{2}^{\prime}(X)=$ $\Omega_{2}^{\prime}(\Phi), F\left(T_{2}\right)=F(G)=\varnothing$, hence $\Omega_{2}(X)=\Omega_{2}(\Phi)$. The case $\Phi=S^{2} \rho_{n}-1+k \rho_{n}$ is similar. $\bar{W} \simeq S_{n}$ acts on $X^{T}$ by reflections, and using 3.8 we find some $G_{x}=$ $S[O(n-2) \times O(2)]$. Then the same type of calculation can be repeated.

(ii) Let $G=S O(n), n=2 m+1, \Phi=\operatorname{Ad}_{G}\left(\right.$ or $\left.S^{2} \rho_{n}-1\right)+k \rho_{n}$. Since the $S O(2 m)$ case is settled, we restrict $X$ to $S O(2 m)$, and by $W_{2}$-invariance of $\Omega_{2}^{\prime}(X)$ it is easily checked that $\Omega_{2}^{\prime}(X)=\Omega_{2}^{\prime}(\Phi)$. It remains to determine $F(T) \sim S^{r}$, or equivalently, $F\left(T_{2}\right) \sim \mathbf{z}_{2} S^{d}$.

Let $\Phi=\operatorname{Ad}_{G}+k \rho_{n}$. Then

$$
\begin{aligned}
\operatorname{dim} X & =r+\# \Omega^{\prime}(X)=d+\# \Omega_{2}^{\prime}(X) \\
& =\operatorname{dim} S O(n)+k n+d \Rightarrow r=k+m+d .
\end{aligned}
$$

Let $S O(2 m)$ (resp. $S O(2 m)^{\prime}$ ) be the upper (resp. lower) standard imbedding into $S O(2 m+1)$. By (i), $F(S O(2 m))=F\left(T_{2} \cap S O(2 m)\right)$, implying

$$
F\left(T_{2}\right)=F\left(T_{2} \cap S O(2 m)\right) \cap F\left(T_{2} \cap S O(2 m)^{\prime}\right)=F(S O(n))=\varnothing,
$$

hence $d=-1, r=k+m-1$ as in the model $\Phi$.

Let $\Phi=S^{2} \rho_{n}-1+k \rho_{n}$. Consider the action of $W_{2}=S_{n}$ on $F\left(T_{2}\right)$. We claim that $S_{n}$ acts effectively as a group generated by reflections, and prove this as follows.

Consider the corank 2 subgroup $T_{2}^{\prime \prime} \subset T_{2}$ given by $\varepsilon_{1}=\varepsilon_{2}=\varepsilon_{3}$, cf. (39). The group $Z_{G}\left(T_{2}^{\prime \prime}\right) / T_{2}^{\prime \prime}=S\left[O(3) \times O(1)^{n-3}\right] / T_{2}^{\prime \prime} \simeq S O(3)$ acts on $Y=F\left(T_{2}^{\prime \prime}\right) \sim \mathbf{z}_{2} S^{d+3}$ with $\Omega_{2}^{\prime}(Y)=\left\{\varepsilon_{1}, \varepsilon_{2}, \varepsilon_{3}\right\}$ and $F\left(D_{2}, Y\right)=F\left(T_{2}\right) \sim \mathbf{z}_{2} S^{d}$. Let $S_{3}=N\left(D_{2}\right) / D_{2}$ be the 2-Weyl group of $S O(3)$. It is not difficult to see that $F(S O(2), Y) \neq \varnothing$ and $\Omega^{\prime}(Y)=\Omega^{\prime}\left(\rho_{3}\right)$ or $\Omega^{\prime}\left(S^{2} \rho_{3}\right)$. From this we infer $F\left(S_{3}, F(T)\right)=F(S O(3), Y)$, and hence also $F\left(S_{n}, F\left(T_{2}\right)\right)=F(S O(n))=\varnothing$. But $\Omega^{\prime}(Y)=\Omega^{\prime}\left(\rho_{3}\right)$ would imply $F\left(S_{3}, F\left(T_{2}\right)\right)=F\left(T_{2}\right)$, and therefore $F\left(S_{n}, F\left(T_{2}\right)\right)=F\left(T_{2}\right)=\varnothing$. However, this is not consistent with the restricted weight patterns $\Omega$ and $\Omega_{2}$ of $X \mid S O(2 m)$. So $\Omega^{\prime}(Y)=\Omega^{\prime}\left(S^{2} \rho_{3}\right)$, and then it is not difficult to see that $S_{3}$ acts faithfully on $F\left(T_{2}\right)$ as reflection group. Since $n \geq 5, S_{n}$ acts faithfully on $F\left(T_{2}\right)$ as reflection group, consequently

$$
S^{-1}=F\left(S_{n}, F\left(T_{2}\right)\right) \sim_{\mathbf{z}_{2}} S^{d-n+1},
$$

and $d=n-2$ as in the model $\Phi$. 
NOTE. The above analysis also gives

$$
\begin{aligned}
F\left(S\left[O\left(l_{1}\right) \times O\left(l_{2}\right) \times \cdots \times O\left(l_{q}\right)\right]\right) \\
\quad=F\left(S_{l_{1}} \times S_{l_{2}} \times \cdots \times S_{l_{q}}, F\left(T_{2}\right)\right) \sim_{\mathbf{z}_{2}} S^{n-2-\Sigma l_{\mathbf{i}}+q},
\end{aligned}
$$

(iii) Finally, let $G=S O(m) \times S O(n), m, n$ odd, $\Phi=\rho_{m} \otimes \rho_{n}+k \rho_{m}+k^{\prime} \rho_{n}$, $F(T) \sim S^{r}, r \geq 0$. We must show $r=k+k^{\prime}, \Omega_{2}^{\prime}(X)=\Omega_{2}^{\prime}(\Phi)$. Let $T$ (resp. $T_{2}$ ) be the standard maximal torus (resp. 2-torus) of $G$.

Note first that $F(T)=F(S O(m-1) \times S O(n-1))$ and the local representation of $S O(m-1) \times S O(n-1)$ is $\Phi_{1}=\rho_{m-1} \otimes \rho_{n-1}+(k+1) \rho_{m-1}+\left(k^{\prime}+1\right) \rho_{n-1}$ (mod trivial). Moreover, the $\mathbf{Z}_{2}$-weight system

$$
\begin{aligned}
\Omega_{2}^{\prime}\left(\Phi_{1}\right)=\left\{\left(\varepsilon_{i} \varepsilon_{j}^{\prime}\right)\right\}+(k+1)\left\{\varepsilon_{i}\right\}+\left(k^{\prime}+1\right)\left\{\varepsilon_{i}^{\prime}\right\} \\
\left(\varepsilon_{i}^{\prime} \text { refers to the second factor } S O(n)\right)
\end{aligned}
$$

is the restriction of $\Omega_{2}^{\prime}(X)$ obtained by putting $\varepsilon_{m}=\varepsilon_{n}^{\prime}=1$. By 2-Weyl group invariance of $\Omega_{2}^{\prime}(X)$, we must have $\Omega_{2}^{\prime}(X)=\Omega_{2}^{\prime}(\Phi)$.

Assume $m \geq 5, n \geq 5$. Then $\Omega_{2}^{\prime}(X) \cap \Delta_{2}(G)=\varnothing$ and this can be shown to imply $F\left(T_{2}\right)=F(G)$, e.g., using [9, Theorem (1.15)]. Therefore, $\Omega_{2}(X)=\Omega_{2}(\Phi)$ and $r=k+k^{\prime}$ follows from counting dimensions. [The conclusion also holds for $n=3$ or $m=3$ (or both), by a different method.]

(E) Determination of orbit types. Here we shall explain how the above results on weight pattern, namely $\Omega(X)=\Omega(\Phi), \Omega_{p}(X)=\Omega_{p}(\Phi)$, can be used to show $O(X)=O(V(\Phi))$, where $\Phi$ is one of the ten basic models of Table I, and $X \sim$ $S^{N}=V(\Phi)$ as before.

First, since $O^{t}(X)$ is an invariant of $\Omega(X)$ (by the torus type algorithm, cf. $\S 3(\mathrm{~B}))$, we have $\mathrm{O}^{t}(X)=\mathrm{O}^{t}(V(\Phi))$. In all cases $\Phi=\operatorname{Ad}_{G}+k \psi_{n}, k>0$, it follows from Lemma 3.5 (or directly from Table I) that the above collection consists of those subtori $S \subset T$ of "standard" type, that is,

$$
S=\theta_{i_{1}}^{\perp} \cap \theta_{i_{2}}^{\perp} \cap \cdots \cap \theta_{i_{q}}^{\perp}, \quad \theta_{i_{j}} \in \Omega^{\prime}\left(\psi_{n}\right) .
$$

Similar results hold for the maximal $\mathbf{Z}_{p}$-tori of isotropy groups.

Observe that $F(G)$ always can be expressed either as $F(T), F(W, F(T)), F\left(T_{2}\right)$ or $F\left(W_{2}, F\left(T_{2}\right)\right)$, and $W$ or $W_{2}$ acts "nicely", cf. (B). If we had $F(G) \neq \varnothing$ (i.e., $X \sim$ pt or $l>0)$, then $O(X) \supset O(V(\Phi))$ would follow by local linearity. However, with $X \sim S^{N}$ and $F(G)=\varnothing$ we still have $F(H, X) \neq \varnothing$ for those maximal groups in $O(\Phi)-\{G\}=O(V(\Phi))$. From case by case considerations one checks that $G_{x} \supset H$ implies $G_{x}=H$, so again by local linearity, $O(X) \supset O(V(\Phi))$.

It remains to show $O(X) \subset O(V(\Phi))$. We shall discuss the cases of $\Phi$ different from \#2, 3 or 10 in Table I. Let $G_{x}$ be an isotropy group; its maximal torus is of "standard" type, and so $G_{x}^{0}=H \subset G(m) \subset G(n)$, where $H$ has maximal rank in $G(m)$, cf. Theorem 2.2. Let us assume rk $H \geq 2$. Now, the restriction $X \mid G(m)$ and $X$ have weight pattern of same type (same number in Table I). Both $X \mid G(m)$ and $V(\Phi) \mid G(m)$ belong to the category of $G(m)$-spaces described in 3.8 , and their Cartan-Weyl actions have the same isotropy types (this is obvious if $G \neq S O$ (odd)). Hence, $X$ and $V(\Phi)$, restricted to $G(m)$, have the same maximal rank isotropy types. It follows that $H \in 0^{\circ}(\Phi)$.

Now it is rather straightforward to show $0^{0}(X)=0^{0}(V(\Phi))$ implies $O(X)=$ $O(V(\Phi))$. As an example, let $G=S U(n), \Phi=\operatorname{Ad}_{G}+k \mu_{n}$, and let $G_{r}^{0}=H \subset$ $S U(m)$, say $H \neq 1$ and $2 \leq m<n, m$ minimal. Assume $G_{x} \neq H$ and choose 
$g \in G_{x} \backslash H$ of prime order, and put $K=\langle H, g\rangle$. Then for some $y \in X$

$$
H \subset K \subset G_{x} \subset S[U(m) \times U(n-m)]=G_{y},
$$

and $F(K, X)=F\left(\mathbf{Z}_{p}, F(H, X)\right) \sim \mathbf{z}_{p} S^{q}$ since $F(H)=F\left(W_{H}, F(T)\right) \sim \mathbf{z} S^{h}$. By Corollary 3.3, for some $G_{z}$ in the slice representation of $G_{y}, G_{z} \supset K, G_{z}^{0}=H$. But this slice representation is identical to the slice representation in the linear model, in particular, the isotropy groups are connected. This contradiction shows $G_{x}=H$.

(F) Concluding remarks. (i) Model \#10 in Table I, with $k+k^{\prime}=0$, is just the isotropy representation of Grassman manifolds. The addition of standard copies $\psi_{m}$ or $\psi_{n}$ causes no more difficulties than in the other models, roughly because this model is also "stable" with respect to restriction to standard subgroups.

(ii) P. Yang [12] analyzes some of the cases corresponding to $k=0$ in Table I. $S U(n)$-manifolds modeled after \#2 in Table I are also discussed in [8, §3]. Technically, case \#3 is somewhat similar to \#2. We can make use of P. Yang's technique to show that $O(X) \supset \mathcal{O}\left(\Lambda^{2} \mu_{n}\right)$ (resp. $\left.O\left(S^{2} \mu_{n}\right)\right)$ also holds for $k>0$. Then the reverse inclusion is established as in (E).

(iii) As noted in Theorem B, for models \#2 or 3 it may not be possible to describe the cohomological structure of the fixed point sets $F(H, X)$ in a nice uniform way. We shall explain this as follows, choosing the model \#2. Let $H=\operatorname{Sp}\left(l_{1}\right) \times \cdots \times$ $\operatorname{Sp}\left(l_{q}\right) \in \mathcal{O}\left(\Lambda^{2} \mu_{n}+k \mu_{n}\right), \sum l_{i}=l \leq n / 2$, and hence $\operatorname{Sp}(1)^{l} \subset H \subset \operatorname{Sp}(l) \subset$ $S U(2 l) \subset S U(n)$. By working with $X \mid S U(2 l)$, the associated "Weyl group" will be $S_{l}=N\left(\operatorname{Sp}(1)^{l}\right) / \operatorname{Sp}(1)^{l}$ acting on $Y=F\left(\operatorname{Sp}(1)^{l}\right)=F\left(T^{\prime}\right)$, where $T^{\prime}$ is a maximal torus of $\operatorname{Sp}(l)$. Moreover, $S_{l}$ as abstract Coxeter group, still acts with parabolic isotropy groups. However, $S_{l}$ does not act as a reflection group, but rather by an action modeled after $\left(S_{l}, \mathbf{R}^{l-1} \oplus \mathbf{R}^{l-1}\right)$, i.e., two copies of the canonical representation. It is not too difficult to show

$$
F(H, X)=F\left(S_{l_{1}} \times \cdots \times S_{l_{q}}, Y\right)=F\left(\mathbf{Z}_{l_{1}} \times \cdots \times \mathbf{Z}_{l_{q}}, Y\right)=F\left(c_{H}, Y\right)
$$

$\left[c_{H}=\right.$ Coxeter element of $\left.W_{H} \subset S_{l}\right]$. In particular, $F(H, X) \sim_{\mathbb{Q}} F(H, V(\tilde{\Phi}))$, $\forall H \in O(X)$ will hold in Theorem B if $X$ is compact acyclic, say. In the sphere case, $X \sim S^{N}$, (41) implies $\chi(F(H, X))=\Lambda\left(c_{H}\right)=1+(-1)^{N}$, where $\chi$ (resp. $\Lambda$ ) is the Euler characteristic (resp. Lefschetz number).

(iv) Consider the 2-parametric representation $\Phi=d \cdot \operatorname{Ad}_{G}+k \psi_{n}$ of $G=S O(n)$, $S U(n)$ or $\operatorname{Sp}(n), d \geq 2$. Then $O(\Phi)=O\left(2 \mathrm{Ad}_{G}\right)$ or $O\left(2 \mathrm{Ad}_{G}+\psi_{n}\right)$ for $k=0$ or $k>0$, respectively, cf. 4.12. In the case $k>0 O(\Phi)$ can be calculated from the case $k=0$ simply by putting $\Phi_{n}=2 \operatorname{Ad}_{G}$ in Lemma 5.2 . Now, with the weight pattern $\Omega^{\prime}(X)=\Omega^{\prime}(\Phi)$, a $G$-manifold $X$ must have orbit structure resembling that of $\Phi$ to a large extent (cf. 4.12(ii) when $\operatorname{rk} G=2$ ); in fact, some special cases seem to indicate that $O(X)=O(\Phi)$ may hold quite generally, e.g., if $X$ is compact. As an example, let $G=S U(n), X \sim S^{N}, N+d(n-1) \equiv 0(\bmod 2)$. Similar to $(41), F(G)=$ $F\left(W_{G}, F(T)\right)=F\left(c_{G}, F(T)\right)$, and hence $\chi(F(G))=\Lambda\left(c_{G}\right)=1+(-1)^{N+d(n-1)}=$ $2, O(X) \supset O(\Phi)$. As with $S_{l}$ in (iii), $W=S_{n}$ will act on $F(T)$ with parabolic isotropy groups, so the maximal rank subfamily of $O(X)$ coincides with that of $O(\Phi)$, namely $O\left(\operatorname{Ad}_{G}\right)$. Furthermore, the lower rank subfamily of $O\left(2 \operatorname{Ad}_{G}+\psi_{n}\right)$ is also quite "regular" and easy to describe; in the present case these groups are the "diagonal blocks" $S\left[\Delta^{k_{1}} U\left(l_{1}\right) \times \Delta^{k_{2}} U\left(l_{2}\right) \times \cdots\right] \subset S\left[U\left(k_{1} l_{1}\right) \times U\left(k_{2} l_{2}\right) \times \cdots\right]$. 
As in the case $n=3$ (cf. proof of 4.11) we find that these are also the lower rank types in $O(X)$.

(v) In conclusion, we point out that although linear actions $\Phi$ are "classified", their orbit structures are, with few exceptions, far from being reasonably well understood. In those few examples with known $O(\Phi)$, the weight pattern $\Omega(\Phi)$ is quite simple. Roughly speaking, as the results of this paper have indicated, these simple weight patterns in the nonlinear case are still sufficiently "strong" as far as full control of $O(X)$ is concerned.

\section{REFERENCES}

1. A. Borel et al., Seminar on transformation groups, Ann. of Math. Studies, no. 46, Princeton Univ. Press, Princeton, N.J., 1961.

2. N. Bourbaki, Groupes et algèbres de Lie, Chapitres IV-VI, Hermann, Paris, 1968.

3. W. C. Hsiang and W. Y. Hsiang, Differentiable actions of compact connected classical groups, I, II, III, Amer. J. Math. 89 (1967), 705-786; Ann. of Math. 92 (1970), 189-223; ibid. 99 (1974), 220-256.

4. W. Y. Hsiang, On the splitting principle and the geometric weight system of topological transformation groups, I, Proc. 2nd Conf. on Compact Transformation Groups, Amherst, Mass., 1971, Lecture Notes in Math., vol. 298, Springer-Verlag, 1972, pp. 334-402.

5. _ Cohomology theory of topological transformation groups, Springer-Verlag, 1975.

6. W. Y. Hsiang and E. Straume, Actions of compact connected Lie groups with few orbit types, J. Reine Angew. Math. 334 (1982), 1-26.

7. __ Actions of compact connected Lie groups on acyclic manifolds with low dimensional orbit space, J. Reine Angew. Math. 369 (1986), 21-39.

8. or projective spaces: Math. Ann. 278 (1987), 71-97.

9. E. Straume, p-weights and their application to regular actions of classical groups, Math. Rep., Univ. of Oslo, 1975.

10. __ Dihedral transformation groups on homology speheres, J. Pure Appl. Algebra 21 (1981), 51-74.

11. _ The topological version of groups generated by reflections, Math. Z. 176 (1981), 429-446.

12. P. Yang, Adjoint type representations of classical groups on homology spheres, Thesis, Princeton Univ., Princeton, N.J., 1974. 